\title{
Holographic model for heavy quarks in anisotropic hot dense QGP with external magnetic field
}

\author{
Irina Ya. Aref'eva, Kristina Rannu and Pavel Slepov \\ Steklov Mathematical Institute, Russian Academy of Sciences, \\ Gubkina str. 8, 119991, Moscow, Russia \\ E-mail: arefeva@mi-ras.ru, rannu-ka@rudn.ru, slepov@mi-ras.ru
}

ABSTRACT: We present a five-dimensional fully anisotropic holographic model supported by Einstein-dilaton-three-Maxwell action. One of the Maxwell fields provides chemical potential; finite chemical potential values are considered. The second Maxwell field serves for anisotropy, representing real spacial anisotropy of the QGP produced in heavy-ion collisions. The third Maxwell field is related to an external magnetic field. Influence of the external magnetic field on the 5-dim black hole solution and the confinement/deconfinement phase diagram, reconstructing the phase transition curves for heavy quarks, is considered. The effect of the inverse magnetic catalyses is revealed and positions of critical end points are found.

KEYwORDS: Holography and quark-gluon plasmas, AdS-CFT Correspondence

ARXIV EPRINT: 2011.07023 


\section{Contents}

1 Introduction 1

2 Model 3

2.1 Metric and EOM 3

2.2 Solution for heavy quarks model 5

3 Thermodynamics $\quad 11$

$\begin{array}{lll}3.1 & \text { Temperature and entropy } & 11\end{array}$

$\begin{array}{lll}3.2 & \text { Free energy and background phase transition } & 15\end{array}$

$\begin{array}{lll}3.3 & \text { Temporal Wilson loops } & 18\end{array}$

4 Conclusions $\quad 24$

\section{Introduction}

Holographic duality provides an effective approach to the study of QCD phase transitions, see for review [1-3]. Holography is a new type of phenomenology. In the context of QCD this means that a phenomenological model, say HQCD (holographical QCD) has to describe QCD at all energy scales - from hight to low scales. The HQCD has to reproduce QCD behaviour at short distances ( $\beta$-function) and Lattice QCD results at large distances (confinement etc.). At an intermediate energy scales and extremal conditions (hight density, or chemical potential) HQCD has to give new theoretical results, that are in agreement with the results of the experimental studies, or predict new results. There are two big groups of models: so-called "top-down" models, related with string models $[4,5]$, and "bottom-up" models. The last ones are phenomenological models that supposed to be found by trial-anderror methods to be able to catch properties of QGP established by different methods and in the same time to be able to predicts the new ones. Typical examples of such phenomena are ones related with non-zero chemical potential and magnetic fields.

Isotropic holographic QCD "bottom-up" models were considered in numerous papers [6]-[55]. These models have been considered to study the confinement/deconfinement phase transition $[6-8,11,13,16-21,23,31,32,35,38,49,53-55]$, as well as the chiral phase transition $[15,22,24,33,34,40,41,44,48,49,51]$ and also to search for quarkyonic phase transition [37]. One of the popular methods for constructing an HQCD within the "bottom-up" approach is a potential reconstruction method for models with Einsteindilaton-Maxwell action. It consists in starting with a given form of metric, which is a deformed form of $A d S_{5}$ [3, 21], and then finding the dilaton potential that supports the given form of the metric. Metric deformation is realized by the warp factor, which in turn determines the blackening function and, consequently, the thermodynamics of the model. 
Choice of the warp factor in the metrics strongly influences the phase transition structure of HQCD. As a guiding principle in the choice of the warp factor one usually uses the agreement with the main features of lattice results. Unfortunately lattice cannot provide us with a full picture of the QCD phase diagram and shows only its individual features. The absence of the general scheme of study for different regimes in QCD is the origin to develop holographic approach to QCD. It occurred that the phase diagram describing heavy quarks can be obtained via deformation of $A d S_{5}$ by wrap factor that is the exponential of a polynomial on holographic coordinate. For the simplest quadratic polynomial $[6,21]$ the model reproduces some features of phase diagram describing heavy quarks, meanwhile to reproduce phase diagram of light quarks one has to use rational functions [11, 28].

It was recognized that it is important to add anisotropy in the holographic theory [14, 56-61] as QGP is an anisotropic media just after the HIC, and an estimation for isotropisation time is about $1-5 \mathrm{fm} / \mathrm{c} \sim 10^{-24} \mathrm{~s}$ [62]. One of motivations to deal with anisotropic models is related with the problem of getting experimental data for the energy dependence of the total multiplicity of particles created in heavy-ion collisions [63]. Isotropic holographic models had not been able to reproduce the experimental multiplicity dependence on energy ([60] and refs therein), therefore to reproduce them anisotropic models with a parameter $\nu$ were considered. It's value of about $\nu=4.5$ gives the dependence of the produced entropy on energy in accordance with the experimental data for the energy dependence of the total multiplicity of particles created in heavy-ion collisions [63].

To deal with anisotropic HQCD one considers Einstein-dilaton-two Maxwell model with additional Maxwell field to support the anisotropy in metrics. Such anisotropic model was considered to reconstruct the picture of the phase transition characteristic of heavy quarks [30] and light quarks [47]. Note that we do not actually calculate quark masses and include them into the action and EOM. Our model just demonstrates effectively the properties of heavy or light quarks' behavior in confinement/deconfinement phase transition. As shown in [32], the model [30] describes smeared confinement/deconfinement phase transitions. This happens since the location on phase diagram of confinement/deconfinement transition line depends on orientation of quark pair in respect to the collision line. That model also indicates the relations of the fluctuations of the multiplicity, i.e. the entanglement entropy, with the background phase transitions [64]. More precisely, the fluctuations of entropy are directly related to the background phase transition, thus pointing to the locations of chiral symmetry breaking. Only in particular cases, when the decay of small black holes to large ones throws out the stable phase to the zone with no dynamical wall, the background phase transition is also the confinement/deconfinement transition. In more general cases, as it has stressed in [52], entanglement entropy is sensitive to mass gap, i.e. the correlation length. Note that anisotropy of the background metric also influences on corresponding jet quenching $[14,65,66]$.

There also is another source of anisotropy - an external magnetic field. Strong magnetic field in the physical 4-dimensional space-time appears in non-central HIC [67-71]. This external magnetic field presents another type of anisotropy and the phenomenon of magnetic catalysis (MC)/inverse magnetic catalysis (IMC) is associated with it. Strong magnetic fields are presented in neutron stars and magnetars $[72,73]$, as well as in the 
early cosmology $[74,75]$. So it is important to take into account this anisotropy in the holographic approach. This anisotropy for improved holographic QCD with Maxwell action has been considered in $[20,25,36,42]$ and for the model with a stack of branes in the Veneziano limit in $[61,76,77]$.

In this paper we present a fully anisotropic holographic model for "heavy quarks", i.e. the model reconstructing the phase diagram characteric for heavy quarks confinement/deconfinement transitions. It is set up by Einstein-dilaton-three-Maxwell action. This model describes two different types of anisotropy: anisotropy for producing the multiplicity dependence on energy (this type of anisotropy is supported by the 2-nd Maxwell field in action) and anisotropy connected with the magnetic field (this type is supported by the 3-rd Maxwell field). The 1-st Maxwell field is introduced to describe finite non-zero chemical potential. We start from a deformed anisotropic metric and recover the dilaton potential and some of kinetic gauge functions from the Einstein-dilaton Maxwell equations. The form of deformation is chosen to reproduce a typical for heavy quarks phase structure, supported by lattice calculations. Deformation of the metric for light quarks is different $[16,28,47]$, and this agrees with the so-called Columbia plot [78-80].

This paper is organised as follows. In section 2 the 5-dim holographic model of hot dense anisotropic QCD in magnetic field is presented. In section 2.1 action and metric to set up the model are introduced and in section 2.2 the corresponding 5-dim BH solution reconstructing heavy quarks model is discussed. Section 3 contains thermodynamic properties of the model in external magnetic field: temperature and entropy behavior (section 3.1), magnetic field influence on the solution properties following from the free energy - the stability of phases and $\mathrm{BH}-\mathrm{BH}$ phase transition picture (BH-BH phase transition curve and critical end points positions) (section 3.2), temporal Wilson loops and crossover regions on the phase diagram with magnetic field (section 3.3). The main conclusions of this investigation and subjects for further research are given in section 4 .

\section{Model}

\section{$2.1 \quad$ Metric and EOM}

We take the action in Einstein frame

$$
\begin{array}{rlrl}
S= & \frac{1}{16 \pi G_{5}} \int d^{5} x \sqrt{-g} \times \\
& \times\left[R-\frac{f_{1}(\phi)}{4} F^{(1)}{ }^{2}-\frac{f_{2}(\phi)}{4} F^{(2)}{ }^{2}-\frac{f_{B}(\phi)}{4} F^{(B)^{2}}-\frac{1}{2} \partial_{\mu} \phi \partial^{\mu} \phi-V(\phi)\right], \\
F_{\mu \nu}^{(1)}= & \partial_{\mu} A_{\nu}-\partial_{\nu} A_{\mu}, \quad \text { i.e. } & A_{\mu}^{(1)}=A_{t}(z) \delta_{\mu}^{0}, \\
F_{\mu \nu}^{(2)}= & q d y^{1} \wedge d y^{2}, \quad \text { i.e. } & F_{y_{1} y_{2}}^{(2)}=q, \\
F_{\mu \nu}^{(B)}= & q_{B} d x \wedge d y^{1}, \quad \text { i.e. } & F_{x y_{1}}^{(B)} & =q_{B},
\end{array}
$$

where $\phi=\phi(z)$ is the scalar field, $f_{1}(\phi), f_{2}(\phi)$ and $f_{B}(\phi)$ are the coupling functions associated with the Maxwell fields $A_{\mu}, F_{\mu \nu}^{(2)}$ and $F_{\mu \nu}^{(B)}$ correspondingly, $q$ and $q_{B}$ are constants 
and $V(\phi)$ is the scalar field potential. Thus (2.1) is the extended version of the action used in $[30,47]$, where we add an external magnetic field $F_{\mu \nu}^{(B)}$.

To consider action (2.1) let us take the ansatz in the following view:

$$
\begin{aligned}
d s^{2} & =\frac{L^{2}}{z^{2}} \mathfrak{b}(z)\left[-g(z) d t^{2}+d x^{2}+\left(\frac{z}{L}\right)^{2-\frac{2}{\nu}} d y_{1}^{2}+e^{c_{B} z^{2}}\left(\frac{z}{L}\right)^{2-\frac{2}{\nu}} d y_{2}^{2}+\frac{d z^{2}}{g(z)}\right], \\
\mathfrak{b}(z) & =e^{2 \mathcal{A}(z)}
\end{aligned}
$$

where $L$ is the AdS-radius, $\mathfrak{b}(z)$ is the warp-factor, $\mathcal{A}(z)$ is related with $\mathfrak{b}(z)$ according (2.4), $g(z)$ is the blackening function, $\nu$ is the parameter of primary anisotropy, caused by nonsymmetry of heavy-ion collision (HIC), and $c_{B}$ is the coefficient of secondary anisotropy related to the external magnetic field $F_{\mu \nu}^{(B)}$. Choice of $\mathcal{A}(z)$ determines the heavy/light quarks version of the model, so we follow previous works and consider $\mathcal{A}(z)=-c z^{2} / 4$ for "heavy quarks" [30] and $\mathcal{A}(z)=-a \ln \left(b z^{2}+1\right)$ for "light quarks" [47].

There are different ways to handle with gauge kinetic functions to include magnetic field in holographic model. Coupling functions $f_{1}$ and $f_{B}$ in Einstein-dilaton-Maxwell-Maxwell action can be different [36, 49] or equal as well [42, 43]. Taking the same gauge function is equivalent to a single Maxwell field on special ansatz, producing both chemical potential and external magnetic field. The choice of $f_{1}$ to realize the linear Regge trajectories for the meson mass spectra was motivated earlier [16, 21, 28]. The gauge function selection occurs at the very beginning and actually leads to a particular problem statement. Note, that the "bottom-up" approach, used in the present and previous works, is characterized by a trialand-error method in choosing an action to support the corresponding ansatz. Therefore our current choice of function can reproduce many phenomenologically expected results (such as phase transitions in $(\mu, T)$ plane), and with acceptable parameters, the structure of the considered holographic model is quite rich. This work does not pretend to be a complete exhaustive description. It is constructed mostly as a versatile setup for further deriving optional variety of particular solutions. Therefore this model needs to satisfy a number of basic requirements, but also keep some degrees of freedom. The purpose of the present work is also to perform a kind of initial calibration of input parameters and specify the significant corrections needed to make the description more realistic and take into account the maximum number of effects possible. Therefore we do not appoint any expression for the coupling function $f_{B}(z)$ and derive while solving the equations of motion.

Putting $f_{1}=f_{B}$ from very beginning and dealing with one Maxwell field is more tricky, and to reproduce the expected phenomenological phase diagram in $(T, \mu, B)$-plane presumably requires more complicated warp-factors and will be the subject of further studies. 
The model's EOM have the form

$$
\begin{gathered}
\phi^{\prime \prime}+\phi^{\prime}\left(\frac{g^{\prime}}{g}+\frac{3 \mathfrak{b}^{\prime}}{2 \mathfrak{b}}-\frac{\nu+2}{\nu z}+c_{B} z\right)+\left(\frac{z}{L}\right)^{2} \frac{\partial f_{1}}{\partial \phi} \frac{\left(A_{t}^{\prime}\right)^{2}}{2 \mathfrak{b} g}- \\
-\left(\frac{L}{z}\right)^{2-\frac{4}{\nu}} \frac{\partial f_{2}}{\partial \phi} \frac{q^{2} e^{-c_{B} z^{2}}}{2 \mathfrak{b} g}-\left(\frac{z}{L}\right)^{\frac{2}{\nu}} \frac{\partial f_{B}}{\partial \phi} \frac{q_{B}^{2}}{2 \mathfrak{b} g}-\left(\frac{L}{z}\right)^{2} \frac{\mathfrak{b}}{g} \frac{\partial V}{\partial \phi}=0, \\
A_{t}^{\prime \prime}+A_{t}^{\prime}\left(\frac{\mathfrak{b}^{\prime}}{2 \mathfrak{b}}+\frac{f_{1}^{\prime}}{f_{1}}+\frac{\nu-2}{\nu z}+c_{B} z\right)=0, \\
g^{\prime \prime}+g^{\prime}\left(\frac{3 \mathfrak{b}^{\prime}}{2 \mathfrak{b}}-\frac{\nu+2}{\nu z}-c_{B} z\right)-2 g\left(\frac{3 \mathfrak{b}^{\prime}}{2 \mathfrak{b}}-\frac{2}{\nu z}-c_{B} z\right) c_{B} z-\left(\frac{z}{L}\right)^{2} \frac{f_{1}\left(A_{t}^{\prime}\right)^{2}}{\mathfrak{b}}=0, \\
2 \mathfrak{b}^{\prime \prime}-\frac{3\left(\mathfrak{b}^{\prime}\right)^{2}}{2 \mathfrak{b}}+\frac{2 \mathfrak{b}^{\prime}}{z}-\frac{4 \mathfrak{b}}{3 \nu z^{2}}\left(1-\frac{1}{\nu}+\left(1-\frac{3 \nu}{2}\right) c_{B} z^{2}-\frac{\nu c_{B}^{2} z^{4}}{2}\right)+\frac{\mathfrak{b}\left(\phi^{\prime}\right)^{2}}{3}=0, \\
\frac{\nu-1}{\nu}+3 g \frac{\nu-1}{\nu}\left(\frac{\mathfrak{b}^{\prime}}{\mathfrak{b}}-\frac{4(\nu+1)}{3 \nu z}+\frac{2 c_{B} z}{3}\right)+\left(\frac{L}{z}\right)^{1-\frac{4}{\nu}} \frac{L q^{2} e^{-c_{B} z^{2} f_{2}}}{\mathfrak{b}}=0, \\
\frac{\mathfrak{b}^{\prime \prime}}{\mathfrak{b}}+\frac{\left(\mathfrak{b}^{\prime}\right)^{2}}{2 \mathfrak{b}^{2}}+\frac{3 \mathfrak{b}^{\prime}}{\mathfrak{b}}\left(\frac{g^{\prime}}{2 g}-\frac{\nu+1}{\nu z}+\frac{2 c_{B} z}{3}\right)-\frac{g^{\prime}}{3 z g}\left(5+\frac{4}{\nu}-3 c_{B} z^{2}\right)+ \\
+\frac{8}{3 z^{2}}\left(1+\frac{3}{2 \nu}+\frac{1}{2 \nu^{2}}\right)-\frac{4 c_{B}}{3}\left(1+\frac{3}{2 \nu}-\frac{c_{B} z^{2}}{2}\right)+\frac{g^{\prime \prime}}{3 g}+\frac{2}{3}\left(\frac{L}{z}\right)^{2} \frac{\mathfrak{b} V}{g}=0,
\end{gathered}
$$

where $^{\prime}=\partial / \partial z$ and

$$
f_{B}=2\left(\frac{z}{L}\right)^{-\frac{2}{\nu}} \mathfrak{b} g \frac{c_{B} z}{q_{B}^{2}}\left(\frac{3 \mathfrak{b}^{\prime}}{2 \mathfrak{b}}-\frac{2}{\nu z}+c_{B} z+\frac{g^{\prime}}{g}\right)
$$

We use the boundary conditions [47]:

$$
\begin{aligned}
A_{t}(0) & =\mu, \quad A_{t}\left(z_{h}\right)=0, \\
g(0) & =1, \quad g\left(z_{h}\right)=0, \\
\phi\left(z_{0}\right) & =0 .
\end{aligned}
$$

where $z_{h}$ is a size of horizon and $z_{0}$ is the integration boundary, $0 \leq z_{0} \leq z_{h}$. Taking $z_{0}=0$ repeats consideration in [28] and $z_{0}=z_{h}$ was used in [30]. The form of the integration boundary $z_{0}=z_{0}\left(z_{h}\right)$ determines the string tension behavior in the model. This aspect was discussed in more details in [47, 54].

Excluding anisotropy and normalizing to the AdS-radius, i.e. putting $L=1, \nu=1$ and $f_{2}=f_{B}=c_{B}=0$ into (2.5)-(2.10), we get the expressions that fully coincide with the EOM (2.12)-(2.16) from [30].

\subsection{Solution for heavy quarks model}

To solve EOM (2.5)-(2.10) we first need to determine the form of the coupling function $f_{1}$. To do this we base on our previous experience. In anisotropic "heavy quarks" [30] and "light quarks" models [47] we used the following expressions:

$$
f_{1 H Q}=z^{-2+\frac{2}{\nu}}, \quad f_{1 L Q}=e^{-c z^{2}-\mathcal{A}(z)} z^{-2+\frac{2}{\nu}} .
$$


To obtain heavy quarks' phase diagram from holography, let us take $\mathfrak{b}=e^{-\frac{c z^{2}}{2}}$ and $f_{1}=z^{-2+\frac{2}{\nu}}$, therefore system $(2.5)-(2.10)$ has the solution ${ }^{1}$

$$
\begin{aligned}
& A_{t}=\mu \frac{e^{\frac{1}{4}\left(c-2 c_{B}\right) z^{2}}-e^{\frac{1}{4}\left(c-2 c_{B}\right) z_{h}^{2}}}{1-e^{\frac{1}{4}\left(c-2 c_{B}\right) z_{h}^{2}}}, \\
& g=e^{c_{B} z^{2}}\left\{1-\frac{\Gamma\left(1+\frac{1}{\nu} ; 0\right)-\Gamma\left(1+\frac{1}{\nu} ; \frac{3}{4}\left(2 c_{B}-c\right) z^{2}\right)}{\Gamma\left(1+\frac{1}{\nu} ; 0\right)-\Gamma\left(1+\frac{1}{\nu} ; \frac{3}{4}\left(2 c_{B}-c\right) z_{h}^{2}\right)}-\right. \\
& -\frac{\mu^{2}\left(2 c_{B}-c\right)^{-\frac{1}{\nu}}}{4 L^{2}\left(1-e^{\left(c-2 c_{B}\right) \frac{z_{h}^{2}}{4}}\right)^{2}}\left(\Gamma\left(1+\frac{1}{\nu} ; 0\right)-\Gamma\left(1+\frac{1}{\nu} ; \frac{3}{4}\left(2 c_{B}-c\right) z^{2}\right)\right) \times \\
& \left.\times\left[1-\frac{\Gamma\left(1+\frac{1}{\nu} ; 0\right)-\Gamma\left(1+\frac{1}{\nu} ; \frac{3}{4}\left(2 c_{B}-c\right) z^{2}\right)}{\Gamma\left(1+\frac{1}{\nu} ; 0\right)-\Gamma\left(1+\frac{1}{\nu} ; \frac{3}{4}\left(2 c_{B}-c\right) z_{h}^{2}\right)} \frac{\Gamma\left(1+\frac{1}{\nu} ; 0\right)-\Gamma\left(1+\frac{1}{\nu} ;\left(2 c_{B}-c\right) z_{h}^{2}\right)}{\Gamma\left(1+\frac{1}{\nu} ; 0\right)-\Gamma\left(1+\frac{1}{\nu} ;\left(2 c_{B}-c\right) z^{2}\right)}\right]\right\}, \\
& f_{B}=-2\left(\frac{z}{L}\right)^{-\frac{2}{\nu}} e^{-\frac{1}{2} c z^{2}} \frac{c_{B} z}{q_{B}^{2}} g\left(\frac{3 c z}{2}+\frac{2}{\nu z}-c_{B} z-\frac{g^{\prime}}{g}\right), \\
& f_{2}=4\left(\frac{z}{L}\right)^{2-\frac{4}{\nu}} e^{-\frac{1}{2}\left(c-2 c_{B}\right) z^{2}} \frac{\nu-1}{q^{2} \nu z} g\left(\frac{\nu+1}{\nu z}+\frac{3 c-2 c_{B}}{4} z-\frac{g^{\prime}}{2 g}\right), \\
& \phi=\int_{z_{0}}^{z} \frac{1}{\nu \xi} \sqrt{4 \nu-4+\left(4 \nu c_{B}+3\left(3 c-2 c_{B}\right) \nu^{2}\right) \xi^{2}+\left(\frac{3}{2} \nu^{2} c^{2}-2 c_{B}^{2}\right) \xi^{4}} d \xi, \\
& V=-\frac{e^{\frac{1}{2} c z^{2}}}{4 L^{2} \nu^{2}}\left\{\left[8(1+2 \nu)(1+\nu)+2(3+2 \nu)\left(3 c-2 c_{B}\right) \nu z^{2}+\left(3 c-2 c_{B}\right)^{2} \nu^{2} z^{4}\right] g-\right. \\
& \left.-\left[2(4+5 \nu)+3\left(3 c-2 c_{B}\right) \nu z^{2}\right] g^{\prime}+2 g^{\prime \prime} \nu^{2} z^{2}\right\} \text {. }
\end{aligned}
$$

Note that the solution (2.16)-(2.21) was obtained for the external magnetic field set as $F_{x y_{1}}^{(B)}=q_{B}$.

To keep in touch not only with our previous model for "heavy quarks" case [30], but also with our holographic description for "light quarks" case [47] we assume $c=0.227$.

As we can see from (2.17), blackening function doesn't actually depend on magnetic "charge" $q_{B}$, but it depends on coupling coefficient $c_{B}$, that characterises influence of the external magnetic field on metric along the $y_{2}$-direction (figure $1 \mathrm{~A}$ ). Positive $c_{B}$ causes the appearance of local maximum of the blackening function under the horizon. This maximum is expressed the brighter the higher the $c_{B}$ value is. Negative $c_{B}$ decreases the blackening function values till the horizon. Putting $c_{B}=0$ brings us back to the previous model without the external magnetic field [30]. The only restriction on parameters' values that we obtain here is $c \neq 2 c_{B}$, since for $c=2 c_{B}$ leads to singular behavior of curvature invariants.

We set $q_{b}=1$ in most of the following calculations.

\footnotetext{
${ }^{1}$ More general forms of $P(z)$ do not admit explicit express for $A_{t}$ and $g$, and will be considered separately.
} 
Comparing plots on figure $1 \mathrm{~A}$ one can notice, that the primary QGP anisotropy, parametrized by $\nu$, suppresses the external magnetic field influence on the blackening function. Moreover, this suppression isn't equal for positive and negative values of coupling coefficient $c_{B}$. For $\nu=4.5$ difference between $c_{B}=0$ and $c_{B}=0.5$ is the smallest comparing to the lesser anisotropy cases, while curves for $c_{B}=1.5$ and $c_{B}=2$ can be hardly distunguished from each other.

On figure $1 \mathrm{~B}$ blackening function depending on chemical potential for $c_{B}=-1$ and primary anisotropy $(1 \leq \nu \leq 4.5)$ is plotted. One can see that the presence of magnetic field doesn't change the picture in general: increasing chemical potential still leads to decreasing of the blackening function values and to the shrinkage of the black hole horizon value as it was in [30]. Increase of primary anisotropy (larger $\nu$ values) enhances the effect of the chemical potential on the blackening function behavior.

On figure $1 \mathrm{C}$ blackening function dependence on $\mu$ for different $c_{B}$ is compared. For larger absolute value of $c_{B}$ chemical potential demonstrates weaker effect of blackening function for small $z$, but stronger influence on the horizon position. In the absence of primary anisotropy ( $\nu=1$ on figure $1 \mathrm{C}$ ) this influence on the horizon position is the most obvious.

Coupling function $f_{B}(2.18)$ depends both on the coefficient $c_{B}$ and the magnetic "charge" $q_{B}$. First of all, $f_{B}$ behavior forces us to limit ourselves to $c_{B}<0$ only, as $c_{B}>0$ makes $f_{B}$ take negative values above the horizon $\left(z<z_{h}\right)$ thus violencing the NEC (figure $2 \mathrm{~A}$ ). For $\nu=1,1.5 f_{B}$ decreases monotonically. Larger primary anisotropy $(\nu=3,4.5)$ leads to more complex function behavior and even to the appearence of local maximum. This effect is more significant for the larger absolute values of $c_{B}$.

Increasing chemical potential makes the coupling function $f_{B}$ with $c_{B}<0$ decrease faster (figure $2 \mathrm{~B}$ ) and reach negative values earlier. Does it mean that large chemical potential forces $f_{B}$ to break NEC at the outer vicinity of horizon? Not really. We should not forget that the horizon itself shifts to smaller $z_{h}$ values because of large chemical potentials. Let us make sure that point, where $f_{B}$ reaches zero, lies inside of horizon anyway as $f_{B}\left(z_{h}\right)$ is still positive.

According to our boundary condition (2.13) $g\left(z_{h}\right)=0$. Therefore expression (2.18) can be simplified as

$$
f_{B}\left(z_{h}\right)=2\left(\frac{z}{L}\right)^{-\frac{2}{\nu}} e^{-\frac{1}{2} c z^{2}} \frac{c_{B} z}{q_{B}^{2}} g^{\prime}\left(z_{h}\right)>0
$$

At first horizon (the one that really matters) blackening function is decreasing, so $g^{\prime}\left(z_{h}\right)<$ 0 . If we also take $c_{B}<0$, their product is positive, all the other multipliers in (2.22) are positive as well, therefore $f_{B}\left(z_{h}\right)>0$ for any negative $c_{B}$ in the $z_{h}$ interval we need.

Until now we only spoke about dependences on $c_{B}$, that characterize the connection between metric and the external magnetic field, but told nothing about the strength of that magnetic field itself. This quality is described by "charge" $q_{B}$.

From (2.18) we see that the coupling function $f_{B}$ depends on the inverse square of the "charge" $q_{B}$. So the larger $q_{B}$ is, the faster $f_{B}$ tends to zero and the longer is in its vicinity (figure $2 \mathrm{C}$ ). 

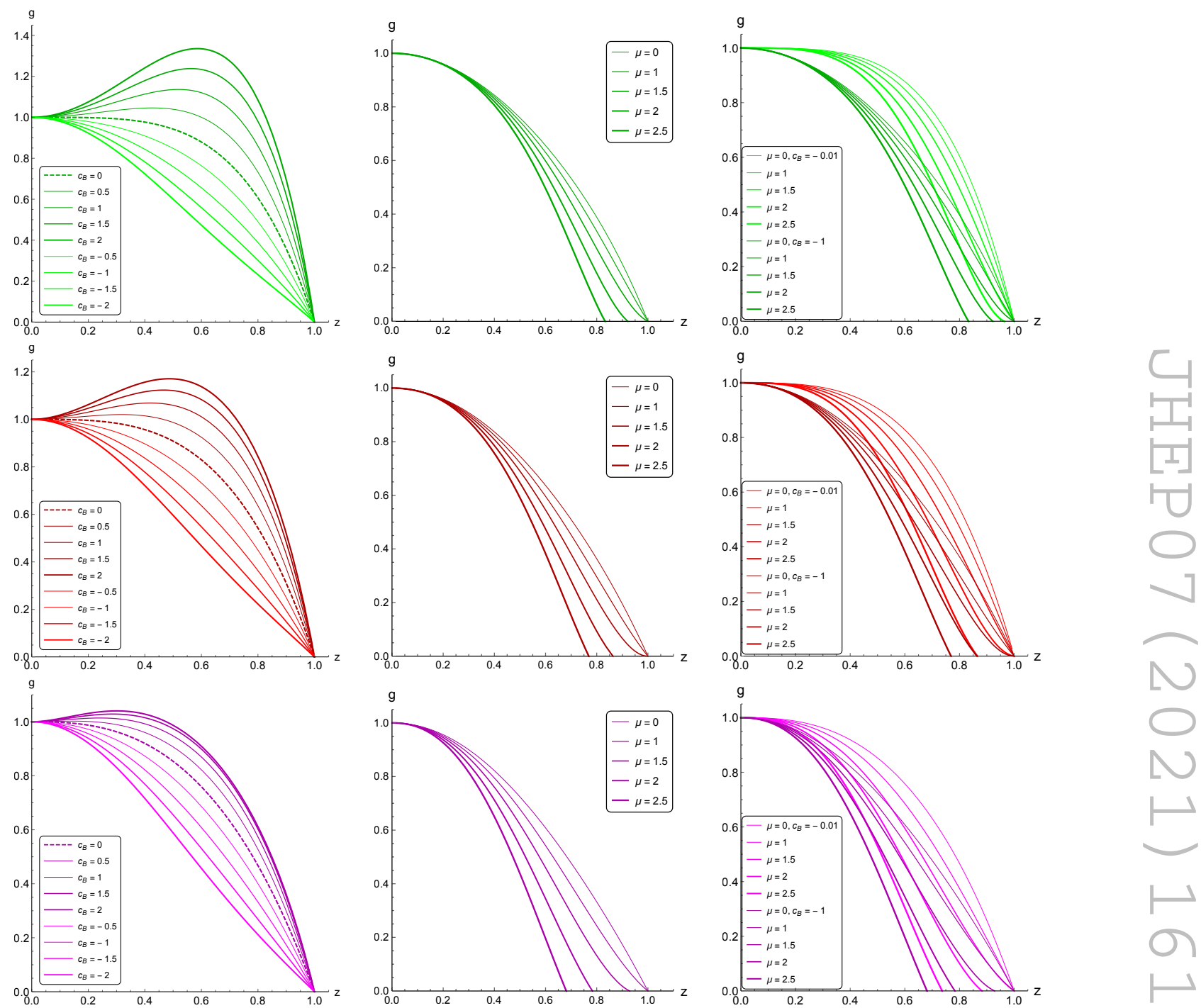

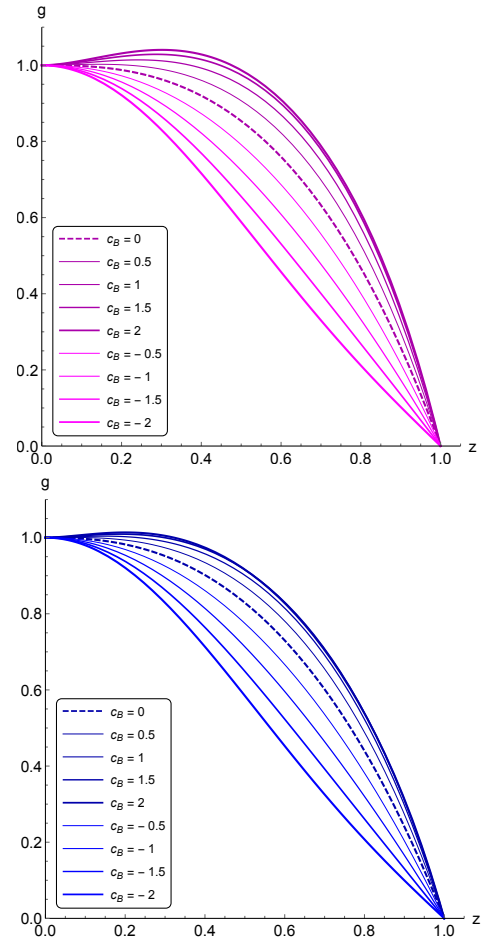

A
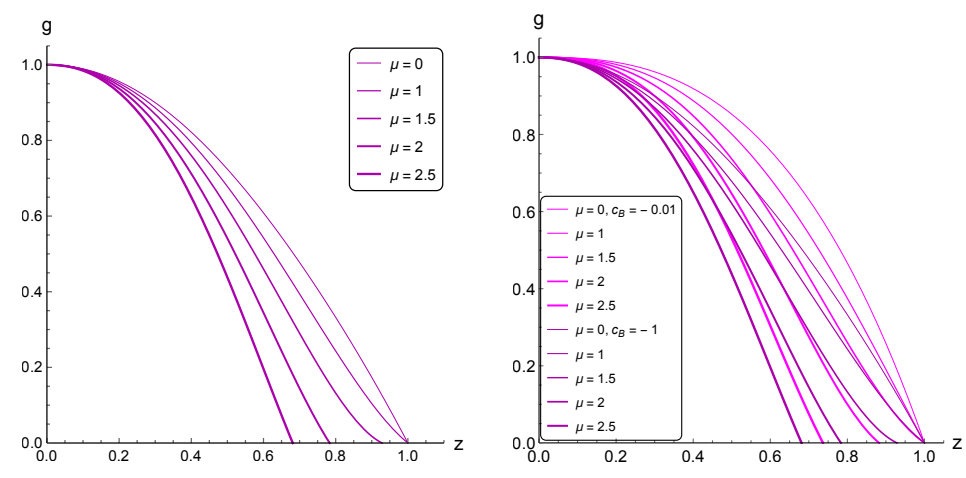

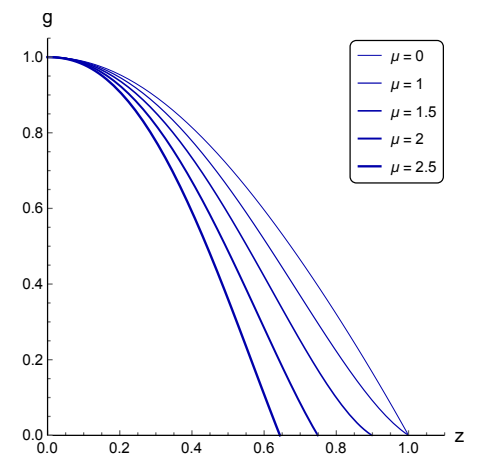

B

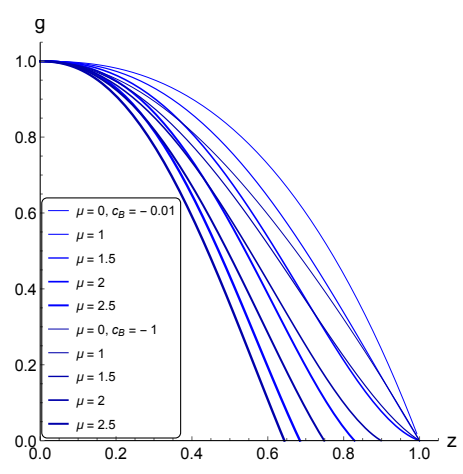

C

Figure 1. Blackening function $g(z)$ for different $c_{B}, \mu=0$ (A), for different $\mu, c_{B}=-1$ (B) and for different $\mu, c_{B}=-0.01$ (light curves) and $c_{B}=-1$ (dark curves) (C); $\nu=1$ (1-st line), $\nu=1.5$ (2-nd line), $\nu=3$ (3-rd line) and $\nu=4.5$ (4-th line), $c=0.227, z_{h}=1$. 


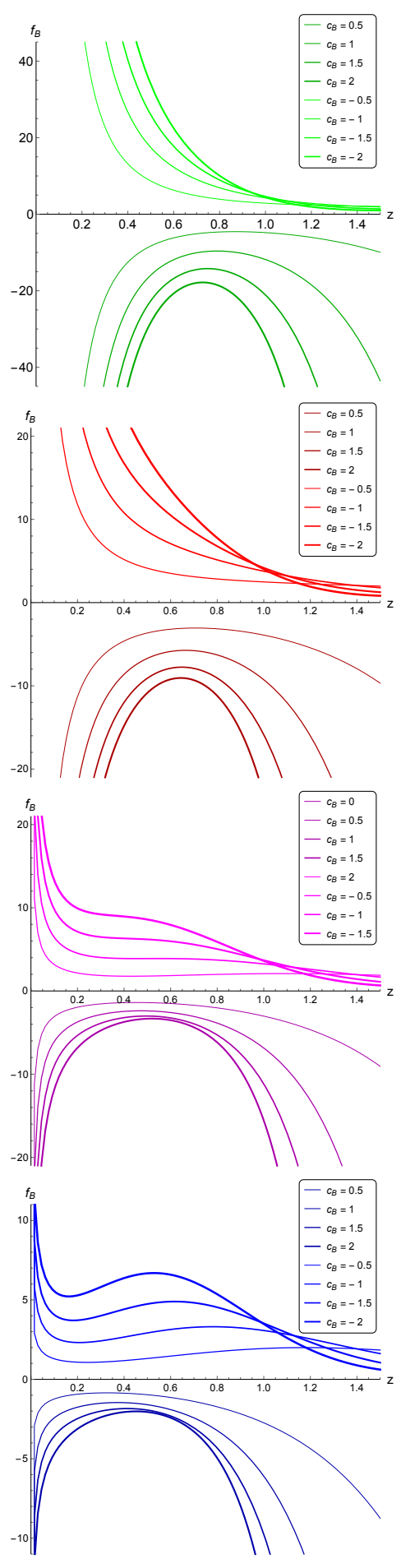

A
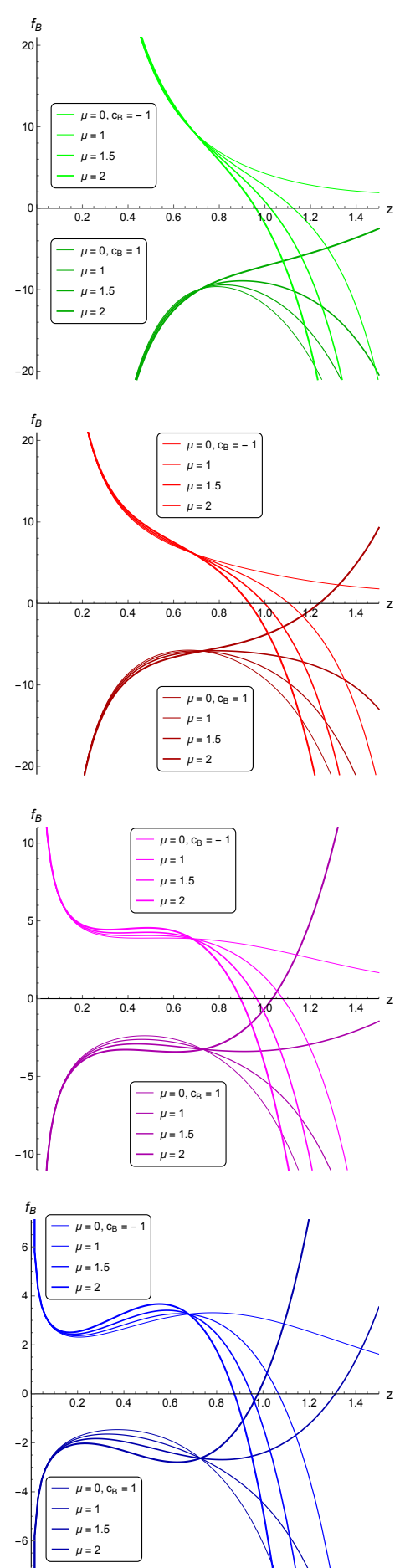

B
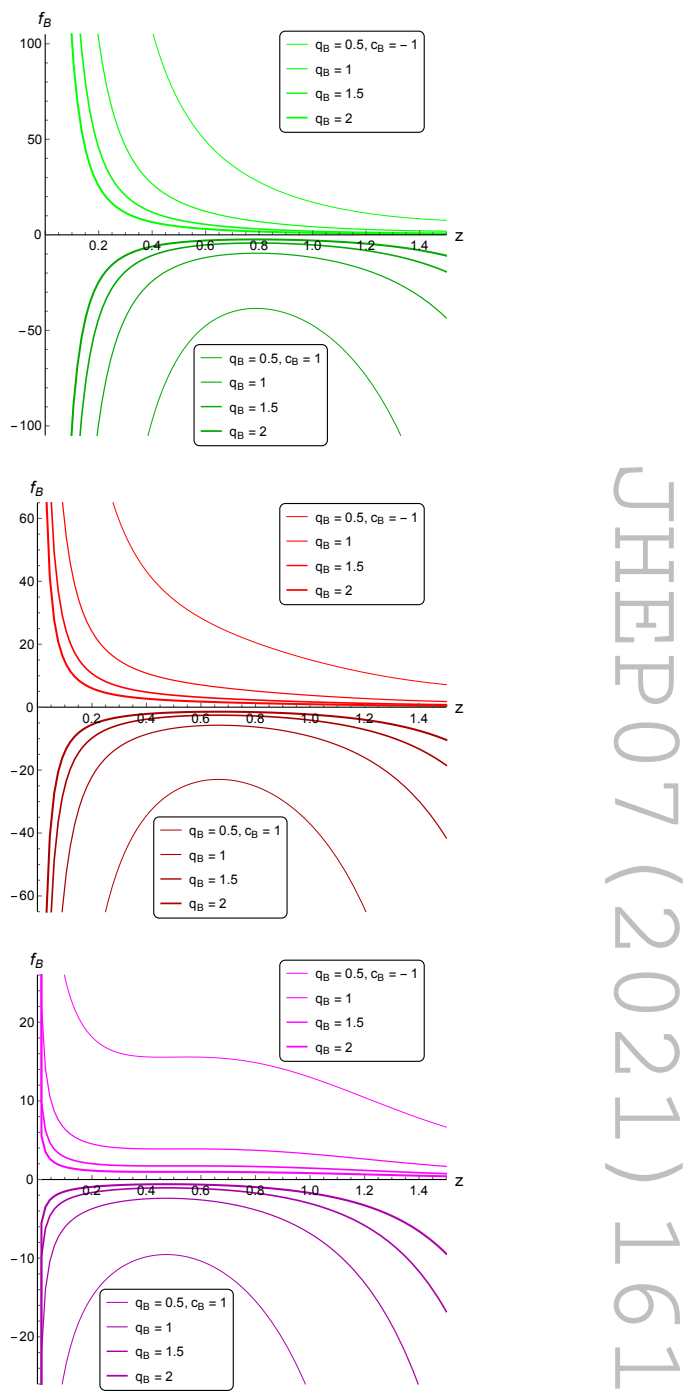

Figure 2. Coupling function $f_{B}(z)$ for different $c_{B}, \mu=0, q_{B}=1$ (A), for different $\mu, c_{B}= \pm 1$, $q_{B}=1$ (B) and for different $q_{B}, \mu=0, c_{B}= \pm 1$ (C); $\nu=1$ (1-st line), $\nu=1.5$ (2-nd line), $\nu=3$ (3-rd line) and $\nu=4.5$ (4-th line), $c=0.227, z_{h}=1$. 


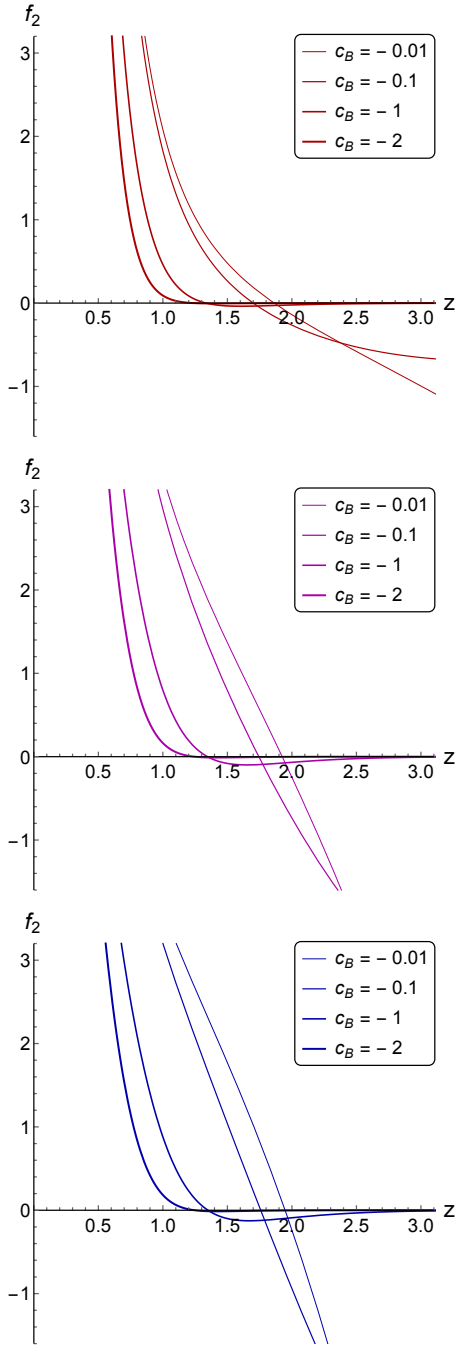

A
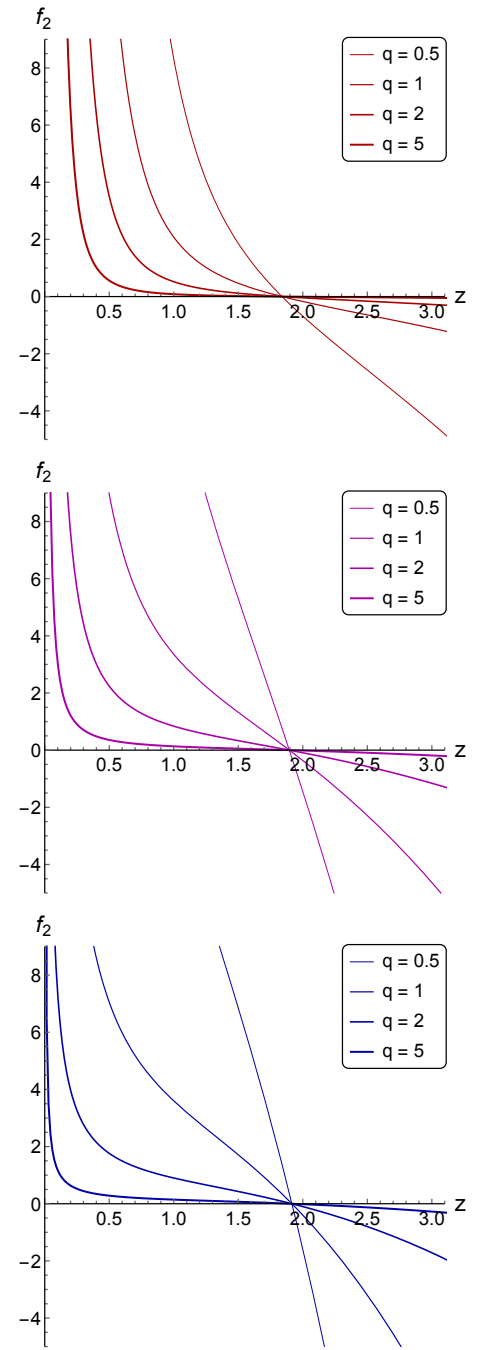

B
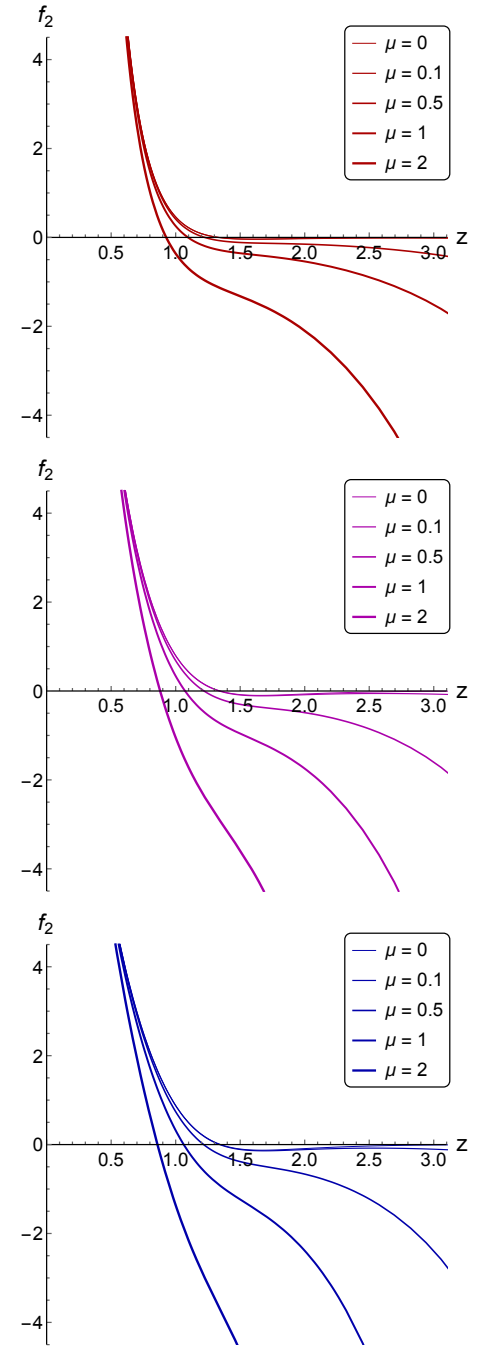

$\mathrm{C}$

Figure 3. Coupling function $f_{2}(z)$ for different $c_{B}, \mu=0, q=1$ (A), for different $q \mu=0, c_{B}=-1$ (B), for different $\mu, c_{B}=-1, q=1(\mathrm{C}) ; \nu=1.5$ (1-st line), $\nu=3$ (2-nd line) and $\nu=4.5$ (3-rd line), $c=0.227, z_{h}=1$.

Let us remind that, unlike the third Maxwell $F_{\mu \nu}^{(B)}$, the second Maxwell field $F_{\mu \nu}^{(2)}$ isn't physical and serves to maintain primary anisotropy, set by parameter $\nu$, of the black hole solution within the holographic approach.

Behavior of the coupling function $f_{2}(2.19)$ in this model is quite similar to its behavior in the absence of external magnetic field [30] (figure 3). Mainly this function descreases monotonically, larger chemical potentials and larger $q$ - "charges" of $F_{\mu \nu}^{(2)}$ - provide faster descrease. These effects are more obvious for larger $\nu$. Case $\nu=1$ doesn't make sense, as $F_{\mu \nu}^{(2)} \equiv 0$ and its coupling function $f_{2}$ do not exist.

For rather large absolute values of coefficient $c_{B}$ (for example $c_{B}=-1$ ) function $f_{2}$ has local minimum, that is more obvious for larger primary anisotropy (figure $3 \mathrm{C}$ ). But this effect is suppressed by chemical potential. 
We can show that $f_{2}$ doesn't break NEC above the horizon like it was done for $f_{B}$ earlier:

$$
f_{2}\left(z_{h}\right)=4\left(\frac{z}{L}\right)^{2-\frac{4}{\nu}} e^{-\frac{1}{2}\left(c-2 c_{B}\right) z^{2}} \frac{\nu-1}{q^{2} \nu z}\left(-g^{\prime}\right) .
$$

At first horizon $g\left(z_{h}\right)=0$ and $g^{\prime}\left(z_{h}\right)<0$. As $f_{2}$ has sense for $\nu>1$ only, all the multipliers in (2.23) are positive, so $f_{2}\left(z_{h}\right)>0$ and NEC isn't broken so far.

Unlike our previous light quarks model [47], this time $z_{0}=0$ is restricted even for $\nu=1$. But we can take $z_{0}$ small enough to maintain the connection to the isotropic limit $[21]$.

Scalar field doesn't depend on $q_{B}$ that characterizes the external magnetic field $F_{\mu \nu}^{(B)}$. Dependence on the coupling coefficient $c_{B}$ is rather slight and weakens with the rise of primary anisotropy, parametrized by $\nu$ (figure $4 \mathrm{~A}$ ).

We are interested in $z \in\left[0 ; z_{h}\right]$, so study the scalar potential $V(\phi)$ for $z<z_{h}$. For larger $z_{h}$ scalar potential reaches lower values $(V(\phi)$-curves become longer). But in the same region of $(\phi, V(\phi))$-plane the scalar potential slightly depends on horizon (figure $4 \mathrm{E}-\mathrm{H}$ ). Coefficient $c_{B}$ characterising coupling with the external magnetic field does not significantly affect scalar potential. For primary isotropic case $\nu=1$ it's influence is comparable with the $z_{h}$ influence (figure $4 \mathrm{E}$ ) and even lesser than the $z_{h}$ influence for $\nu \neq 1$ (figure $4 \mathrm{~F}-\mathrm{H}$ ). In this interval the change in the scalar potential associated with the chemical potential is so small that it can't be depicted on this scale. Therefore we can see that the primary anisotropy is the strongest factor affecting the scalar potential, while the influence of magnetic field and chemical potential are negligible.

For completeness we also present the dependence of kinetic factors $f_{B}$ and $f_{2}$ on the dilaton field $\phi$ (figure 5). Both functions display different behavior for different $z_{h}$ near the $z=z_{h}$ and therefore near $\phi=\phi\left(z_{h}\right)$ (figure 5 1-st line). Curves for different $z_{h}$ naturally have different lengths. As will be seen in the next section, we actually need $c_{B} \in(-0.01 ; 0), \mu \in[0 ; 0.06]$ for $\nu=1$ and $c_{B} \in(-0.02 ; 0), \mu \in[0 ; 0.3]$ for $\nu=4.5$. On figure $5 \mathrm{~A}, \mathrm{~B}$ (2-nd line) we see, that the $f_{B}$-curves for different chemical potentials within these actual intervals can't be distinguished from each other. For $f_{2}$ dependence on the chemical potential is more noticeable, but still negligible (figure $5 \mathrm{C}$ ). Also the function $f_{2}$ almost doesn't depend on magnetic field. Function $f_{B}$ does depend on $c_{B}$, that enters the equation (2.18) as a factor and via the blackening function $g(z)$. This effect is due to the fact, that in this model coupling funtion $f_{B}$ was derived from the EOM as a solution.

\section{Thermodynamics}

\subsection{Temperature and entropy}

For the metric (2.3) and the warp-factor $f_{1 H Q}=z^{-2+\frac{2}{\nu}}(2.15)$ temperature and entropy can be written as:

$$
T=\left.\frac{\left|g^{\prime}\right|}{4 \pi}\right|_{z=z_{h}}, \quad s=\frac{1}{4}\left(\frac{L}{z_{h}}\right)^{1+\frac{2}{\nu}} e^{-\frac{1}{4}\left(3 c-2 c_{B}\right) z_{h}^{2}}
$$




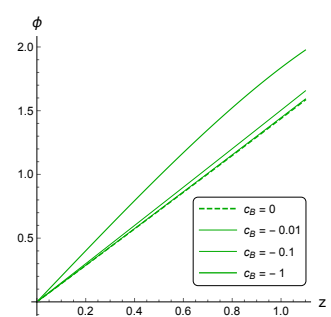

$\mathrm{A}$

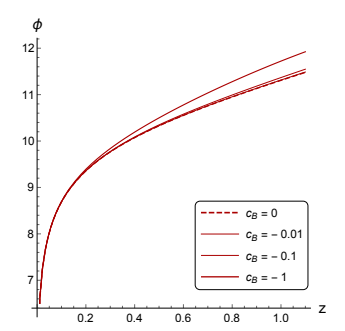

B

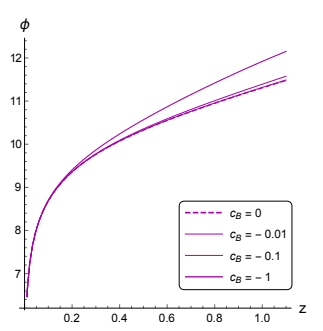

C

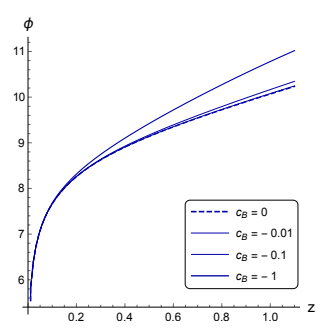

$\mathrm{D}$
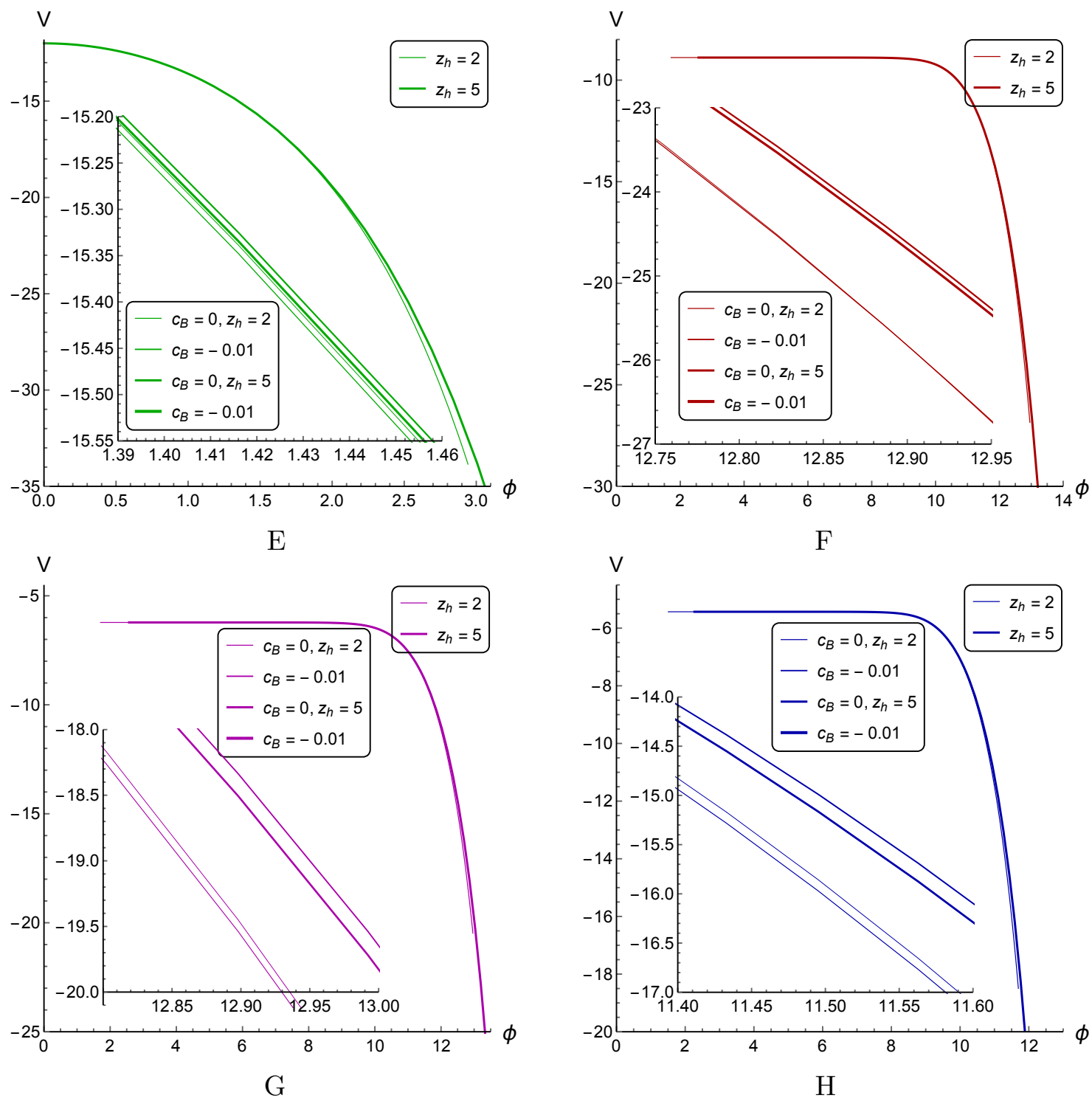

Figure 4. Scalar field $\phi(z)$ for different $c_{B}$ for $\nu=1$ (A), $\nu=1.5(\mathrm{~B}), \nu=3(\mathrm{C}), \nu=4.5$ (D); scalar potential $V(\phi)$ for different $c_{B}$ and $z_{h}$ for $\nu=1(\mathrm{E}), \nu=1.5(\mathrm{~F}), \nu=3(\mathrm{G}), \nu=4.5(\mathrm{H})$ $\mu=0.1, c=0.227$. 

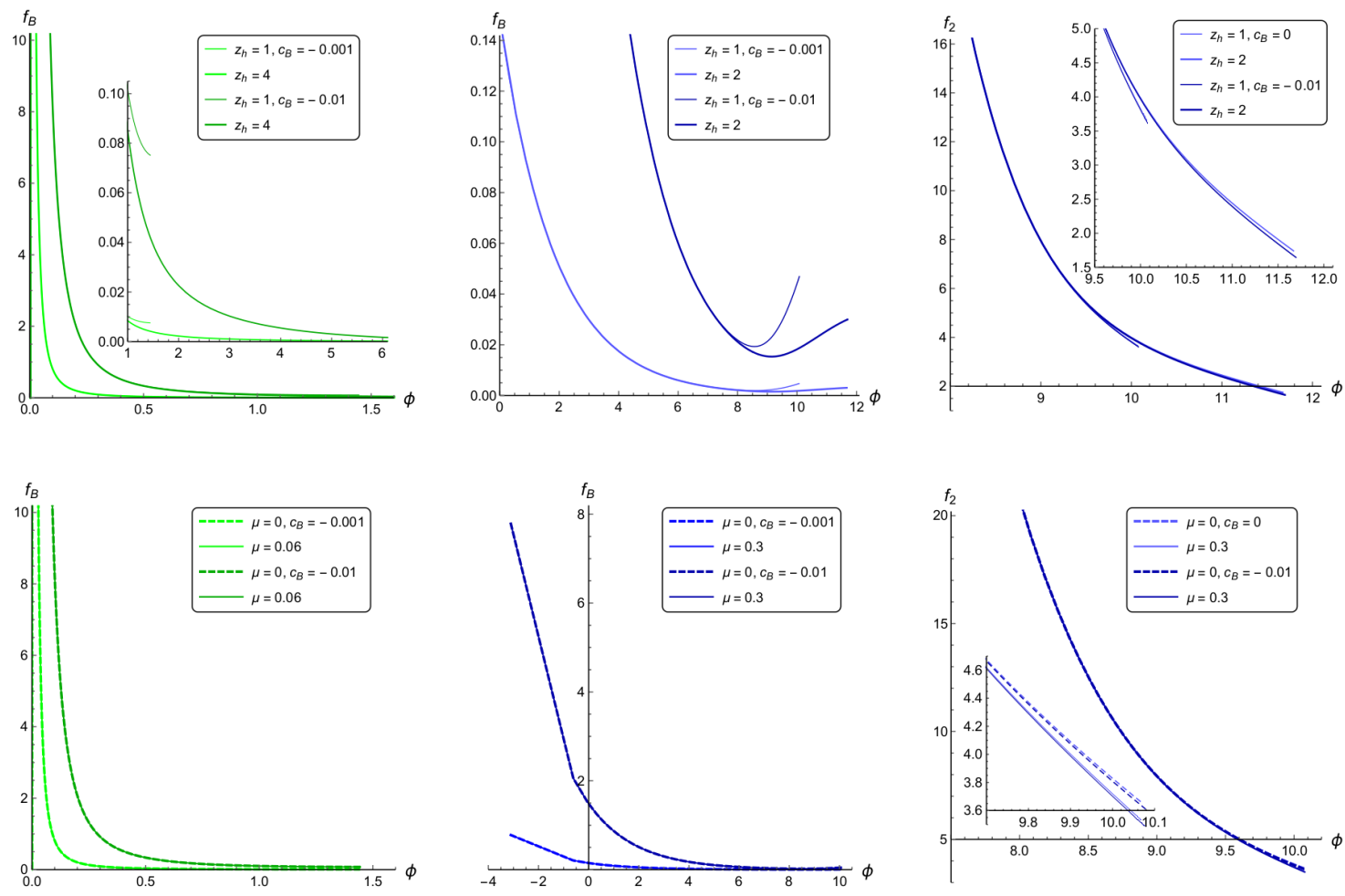

A

$\mathrm{B}$

C

Figure 5. Coupling function $f_{B}(\phi)$ for $c_{B}=-0.001,-0.01, \nu=1,(\mathrm{~A}), \nu=4.5(\mathrm{~B})$ and coupling function $f_{2}(\phi)$ for $c_{B}=0,-0.01(\mathrm{C})$ for $\mu=0$ and different horizons (1-st line) $z_{h}=1,4(\mathrm{~A})$ and $z_{h}=1,2(\mathrm{~B}, \mathrm{C})$ and for $z_{h}=1$ and different chemical potentials (2-nd line) $\mu=0,0.06(\mathrm{~A})$ and $\mu=0,0.3(\mathrm{~B}, \mathrm{C})$.

On figure $6 \mathrm{~A}$ we see, that temperature is quite sensible to $c_{B}$ even for zero chemical potential. Function $T\left(z_{h}\right)$ is multivalued for $c_{B}>-0.02 \div-0.01$ (for different $\nu$ ). For larger anisotropy the ambiguity of temperature is preserved till larger absolute value of coupling coefficient $c_{B}$.

Temperature dependence on chemical potential in the presence of external magnetic field is similar to the previous model [30] (figure 6 C). For larger anisotropy the ambiguity of temperatue is preserved for larger $\mu$ values: till $\mu \approx 0.05$ for $\nu=1$ vs $\mu \approx 0.3$ for $\nu=4.5$.

Behavior of entropy as function of temperature for different values of the coupling coefficient $c_{B}$ (figure $6 \mathrm{~B}$ ) and chemical potential $\mu$ (figure $6 \mathrm{D}$ ) convinces us of the conclusions drawn above. Therefore the confinement/deconfinement phase transition provided by the background (that we called a Hawking-Page-like phase transition previously [30, 32, 47, 55]) in the presence of magnetic field significantly depends on the coupling coefficient $c_{B}$. If the connection of metric with the external magnetic field is too strong $\left(c_{B}<-0.02 \div-0.01\right.$ for different $\nu$ ), the background phase transition shouldn't happen at all. Note, that this process doesn't generally depend on the strength of the field $F_{\mu \nu}^{(B)}$ itself, as both temperature and entropy functions do not contain "charge" $q_{B}$ explicitly. So the coupling coefficient $c_{B}$ turns out to be the parameter that can be fitted via background phase transition. 

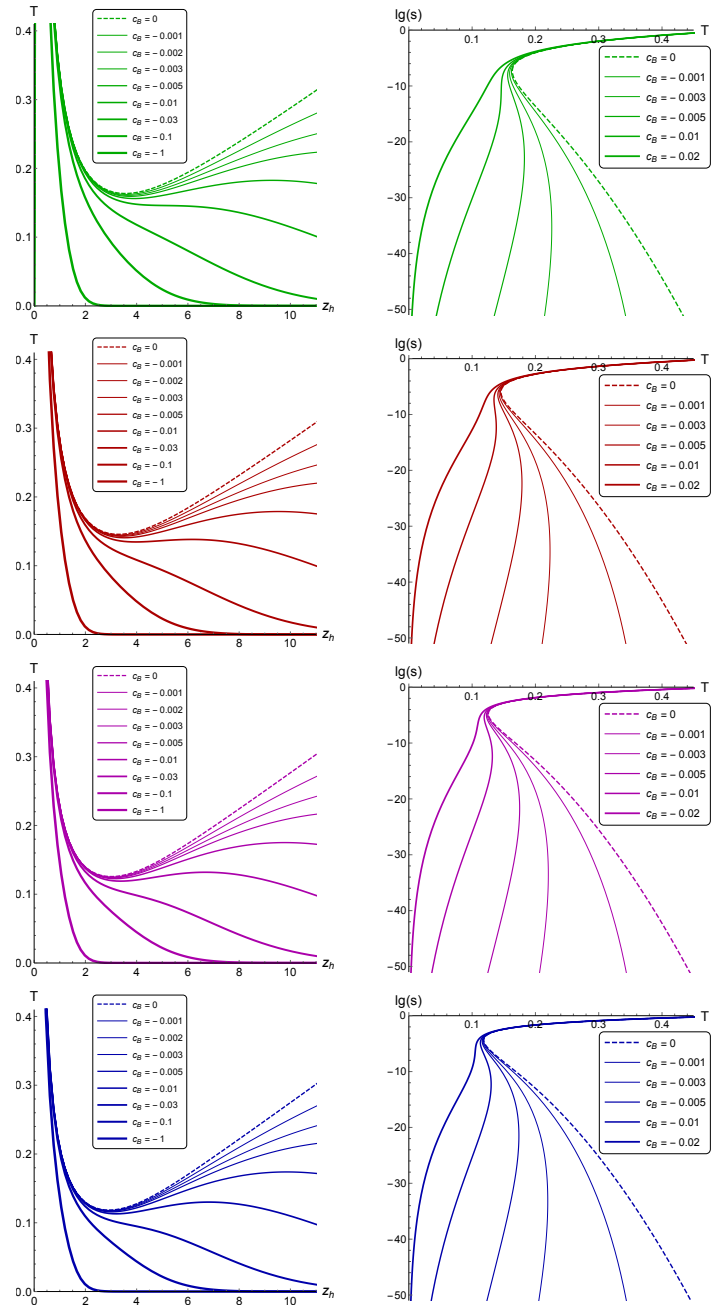

$\mathrm{A}$
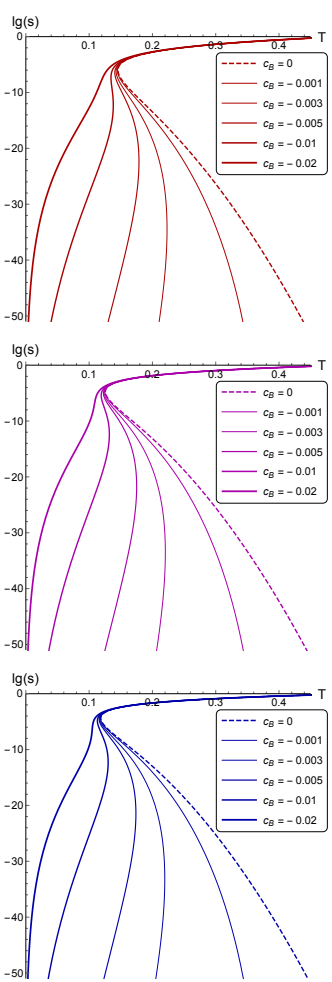

B
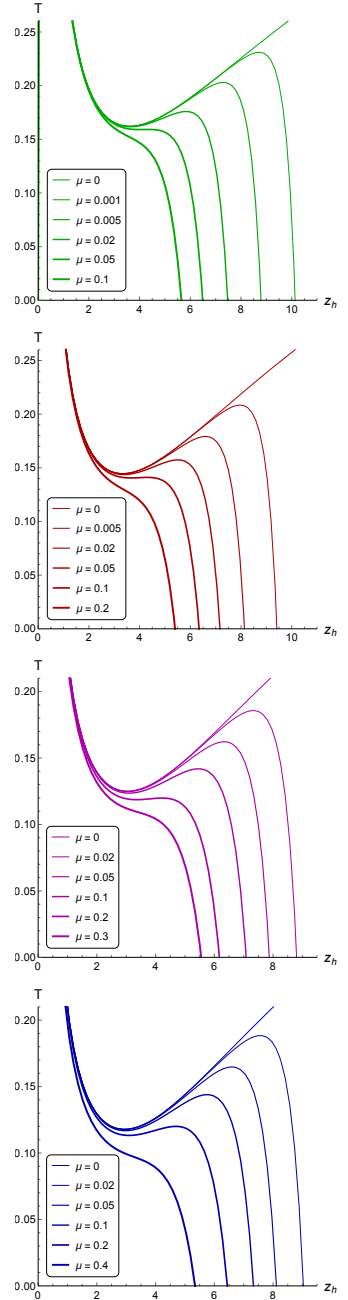

C
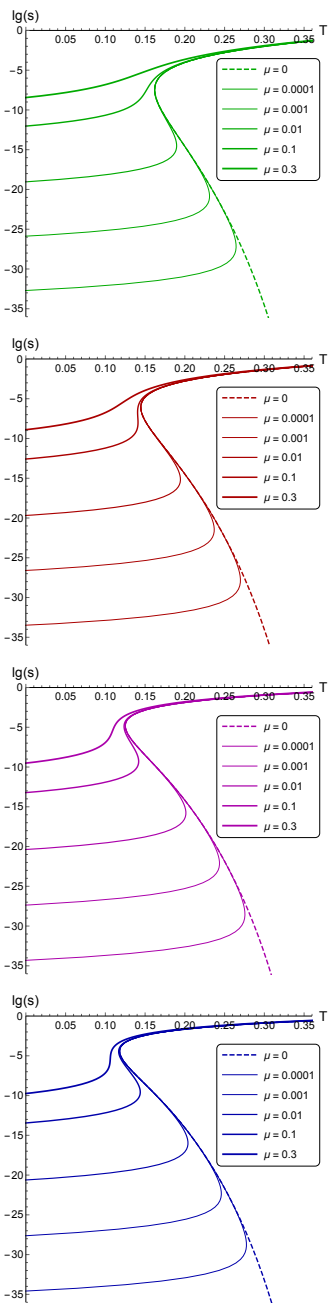

$\mathrm{D}$

Figure 6. Temperature $T\left(z_{h}\right)(\mathrm{A})$ and entropy $s(T)$ in logarithmic scale (B) for different $c_{B}, \mu=0$; temperature $T\left(z_{h}\right)(\mathrm{C})$ and entropy $s(T)$ in logarithmic scale (D) for different $\mu, c_{B}=-0.001 ; \nu=1$ (1-st line), $\nu=1.5$ (2-nd line), $\nu=3$ (3-rd line) and $\nu=4.5$ (4-th line), $c=0.227$.

To ensure that BH-BH transition caused by three-valued temperature function is a phase transition let us consider density $\rho$, that is a coefficient in $A_{t}$ expansion:

$$
\begin{aligned}
A_{t} & =\mu-\rho z^{2}+\cdots=\mu+\frac{\left(c-2 c_{B}\right) \mu z^{2}}{4\left(1-e^{\frac{1}{4}\left(c-2 c_{B}\right) z_{h}^{2}}\right)}+\ldots, \\
\rho & =-\frac{\left(c-2 c_{B}\right) \mu}{4\left(1-e^{\frac{1}{4}\left(c-2 c_{B}\right) z_{h}^{2}}\right)}
\end{aligned}
$$

On figure $7 \rho / \mu$ ratio as a function of temperature for primary isotropic solution $(\nu=1$, figure $7 \mathrm{~A})$ and anisotropic solution $(\nu=4.5$, figure $7 \mathrm{~B})$ are plotted. Vertical arrows show the BH-BH transition direction. Function $\rho / \mu$ is a three-digit function of $T$ as expected, and we can see that collapse from small black holes (larger $z_{h}$ ) to the large ones (smaller 


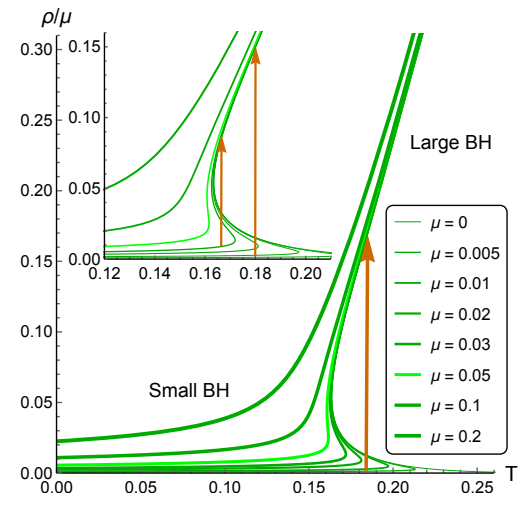

A

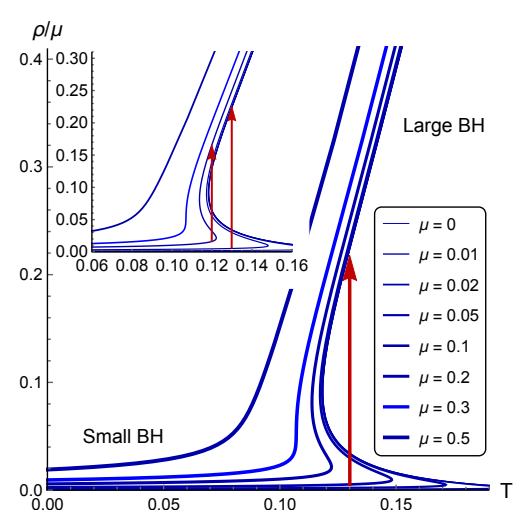

B

Figure 7. Density $\rho / \mu(T)$ for different $\mu$ for $\nu=1$ (A) and $\nu=4.5(\mathrm{~B}) ; c=0.227, c_{B}=0$.

$\left.z_{h}\right)$ is accompanied by a sharp rise of the density for any appropriate chemical potential. On figure $7 c_{B}=0$, but for $c_{B}$ close to zero enough to preserve ambiguity of $T\left(z_{h}\right)$-function density curves will differ from the zero magnetic field case negligibly.

\subsection{Free energy and background phase transition}

To get $\mathrm{BH}-\mathrm{BH}$ phase transition, that originates from the model background, we need to consider the free energy as a function of temperature. According the first law of thermodynamics, the differential of the free energy at fixed volume is defined as $d F=-s d T-\rho d \mu-M d B$, where $\rho$ is the baryon density, $M$ represents the magnetization associated to $B$. For the fixed chemical potential $\mu$ and magnetic field $B$, the free energy can be evaluated by the following integral, cf. [7, 30, 42, 61]

$$
F=-\int s d T=\int_{z_{h}}^{\infty} s T^{\prime} d z
$$

where we have normalized the free energy to vanish at $z_{h} \rightarrow \infty$.

The free energy behavior provides us with the BH-BH (Hawking-Page-like or BB) phase transition coming from the model background. On figure 8 different values of $\Delta F_{B H}(T, \mu)$ are shown. Vertical lines show the chosen temperature $T=0.164$ (figure $8 \mathrm{~A}, \mathrm{~B}$ ) or $T=$ 0.122 (figure $8 \mathrm{C}$ ). It is orange for $\nu=1$ (figure $8 \mathrm{~A}$ ) and red for $\nu=4.5$ (figure 8B,C). Here all quantities are in $\mathrm{GeV}$.

In the absence of magnetic field the free energy difference between two states of the transition is the distance between two red points located on the orange temperature vertical for $\nu=1$ (figure $8 \mathrm{~A}$ ) and between two magenta points located on the red temperature vertical for $\nu=4.5$ (figure $8 \mathrm{~B}, \mathrm{C}$ ). For $c_{B}=-0.001$ the free energy difference is the distance between two dark red points on the same temperature verticals. But on scale of figure $8 \mathrm{~B}$ curves for $c_{B}=0$ and $c_{B}=-0.001$ almost coincide, so red and magenta points are rather close to each other on this plot.

It is interesting to calculate the decay rate of unstable configuration. Computation of nucleation rate of bubbles of stable vacuum in unstable vacuum has been performed in [85], 


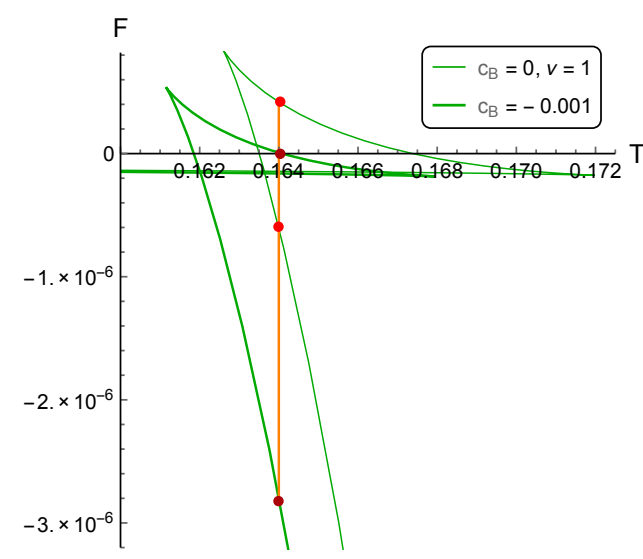

A

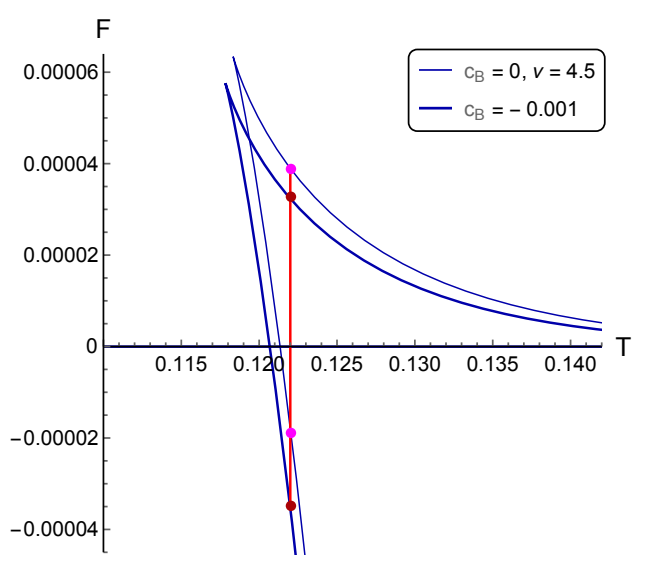

B

Figure 8. Free energy difference $\Delta F(T)$ for $c_{B}=0$ and $c_{B}=-0.001, \nu=1$ (A) and $\nu=4.5$ (B); $c=0.227, \mu=0.03$ and $T=0.164$. Free energy difference and $T$ are in units of $\mathrm{GeV}$.

where authors have found the bounced configurations interpolating between the vacua in Witten-Sakai-Sugimoto model $[4,5]$.

On figure $9 \mathrm{BH}-\mathrm{BH}$ transition lines for different values of primary and secondary anisotropy are presented. Figure $9 \mathrm{~A}-\mathrm{D}$ display the families of curves representing $\mathrm{BH}-\mathrm{BH}$ phase transition lines for different values of primary anisotropy parameter, i.e. figure $9 \mathrm{~A}$ for $\nu=1$, figure $9 \mathrm{~B}$ for $\nu=1.5$, figure $9 \mathrm{C}$ for $\nu=3$ and figure $9 \mathrm{D}$ for $\nu=4.5$.

First of all, larger absolute values of the coupling coefficient $c_{B}$ lead to decrease of the transition temperature, so the effect of the inverse magnetic catalysis takes place. Besides, the length of the Hawking-Page line also decreases and eventually degenerates. However primary anisotropy weakens these effects, so $\mathrm{BH}-\mathrm{BH}$ phase transition is preserved for larger absolute values of the coupling coefficient. For example, background phase transition still exists for $c_{B}=-0.018$ and $\nu=4.5$ (figure 9D), whereas for $c_{B}=-0.001$ and $\nu=1$ no such curve can be plotted (figure $9 \mathrm{~A}$ ).

On figure $9 \mathrm{E}$ all the $\mathrm{BH}-\mathrm{BH}$ phase transition lines are combined into one plot. One can see, that the distance between the lines for the same pairs of $c_{B}$-values shrinks for larger $\nu$, while their length along the $\mu$-axis, on the contrary, grows. Both anisotropy parameters $\nu$ and $c_{B}$ lower the transition temperature, but other their effects on the background phase transition picture can be considered the opposite.

We can also consider positions of the "free ends" of the BH-BH phase transition lines (let us call it "critical end-points for heavy quarks", $\mathrm{CEP}_{H Q}$ ). On figure 10 these positions are depicted on $T-\mu$ (figure $10 \mathrm{~A}$ ), $\mu-c_{B}$ (figure $10 \mathrm{~B}$ ) and $T-c_{B}$ (figure $10 \mathrm{C}$ ) planes. For $\nu=1$ temperature of the $\mathrm{CEP}_{H Q}$ falls relatively quickly with the $c_{B}$ absolute value growth, while for $\nu=4.5$ this temperature is almost constant (figure $10 \mathrm{~A}, \mathrm{C}$ ). On the contrary, chemical potential of the $\mathrm{CEP}_{H Q}$-points falls more for larger primary anisotropy, especially quickly decreasing near the $\mu=0$. On figure 11 the $3 \mathrm{D}$-plots for the $\mathrm{CEP}_{H Q}$ positions depending on coupling coefficient $c_{B}$, chemical potential and temperaure for different values of primary anisotropy are displayed. 


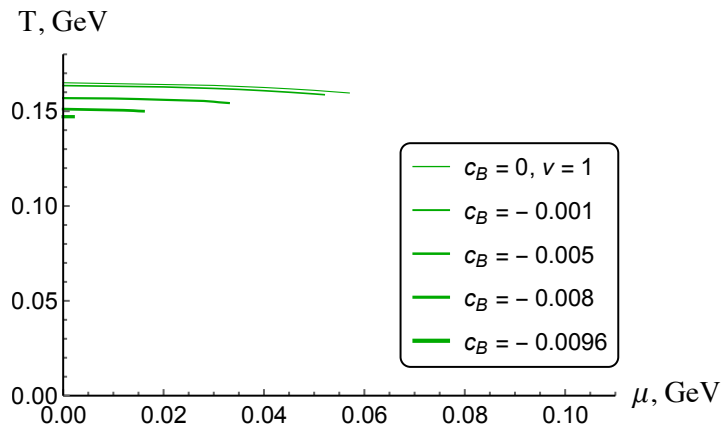

A

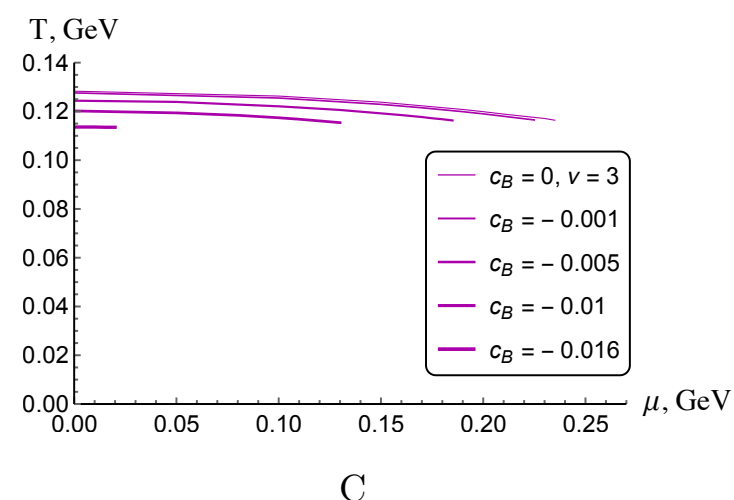

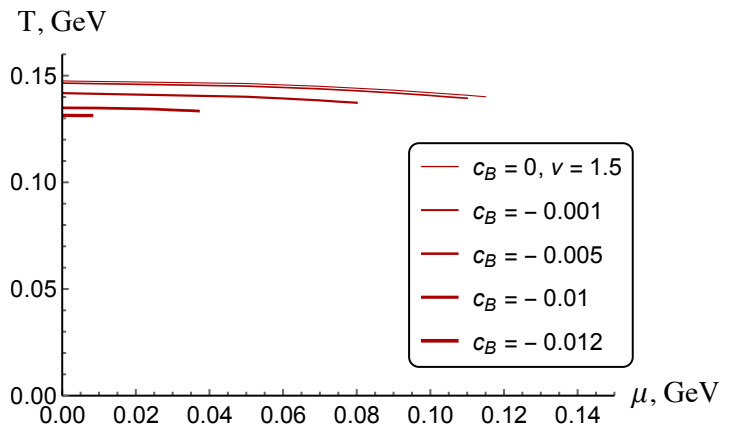

$\mathrm{B}$

$\mathrm{T}(\mu): \mathrm{L}=1, v=4.5, \mathrm{c}=0.227, c_{B}=0$

$\mathrm{T}, \mathrm{GeV}$

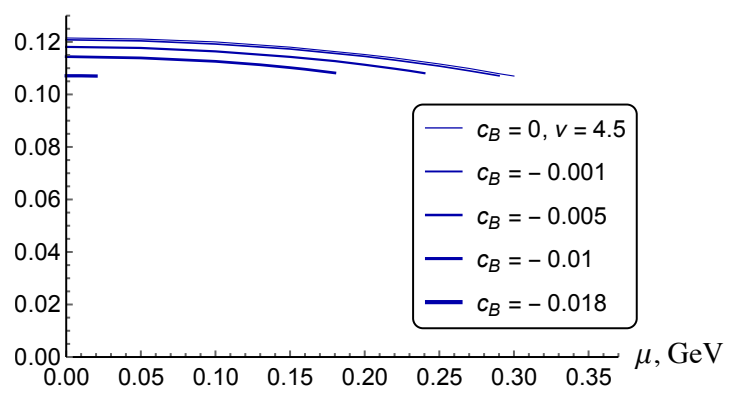

$\mathrm{D}$

$\mathrm{T}, \mathrm{GeV}$

$=\left[\begin{array}{c|c}-c_{B}=0, v=1 \\ -c_{B}=-0.001 \\ -c_{B}=-0.005 \\ -c_{B}=-0.008 \\ -c_{B}=-0.0096\end{array}\right.$

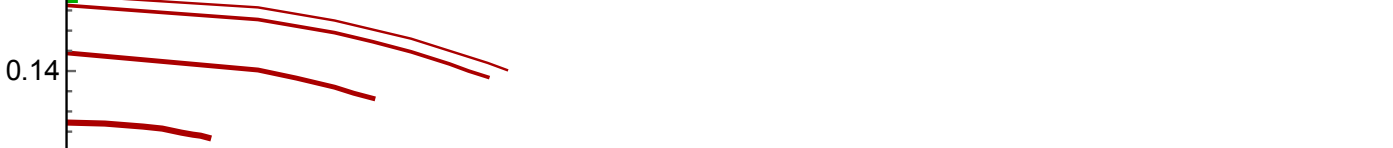

$0.13=$

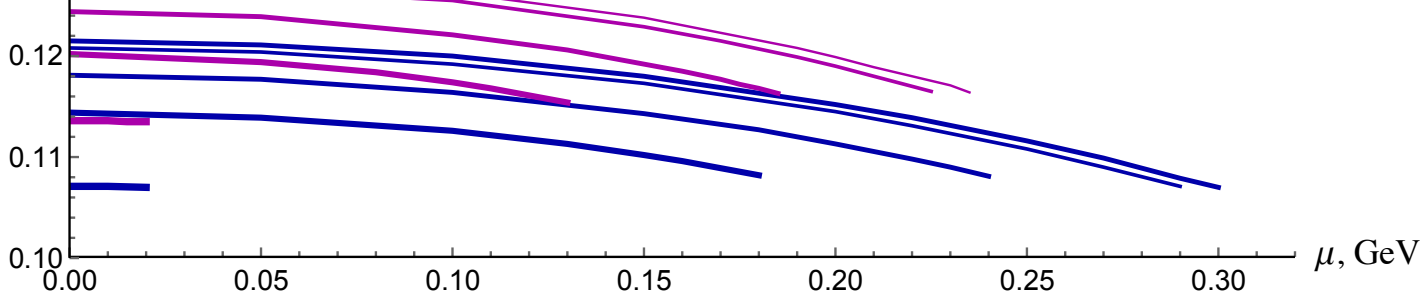

$\mathrm{E}$

Figure 9. Background phase transition lines depending on $c_{B}$ for $\nu=1$ (A), $\nu=1.5$ (B), $\nu=3$ $(\mathrm{C}), \nu=4.5(\mathrm{D})$ and all of them together $(\mathrm{E}) ; c=0.227$. 


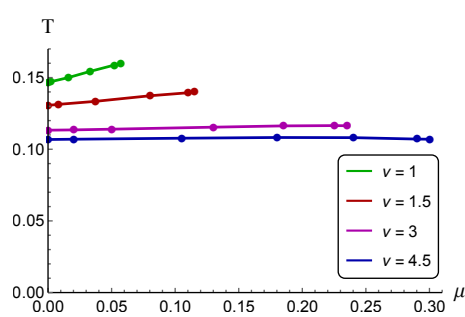

A

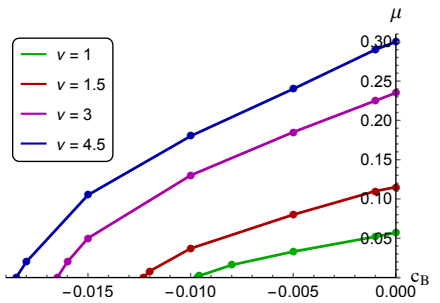

B

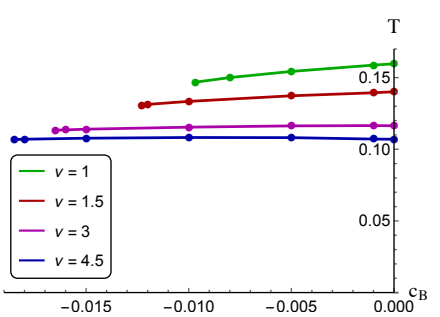

C

Figure 10. Position of critical end-points $\mathrm{CEP}_{H Q}$ for heavy quarks model depending on $\mu$ and $T$ $(\mathrm{A}), c_{B}$ and $\mu(\mathrm{B}), c_{B}$ and $T(\mathrm{C})$ for $\nu=1,1.5,3,4.5, c=0.227$. $T$ and $\mu$ are in units of GeV, and $c_{B}$ of $\mathrm{GeV}^{2}$.

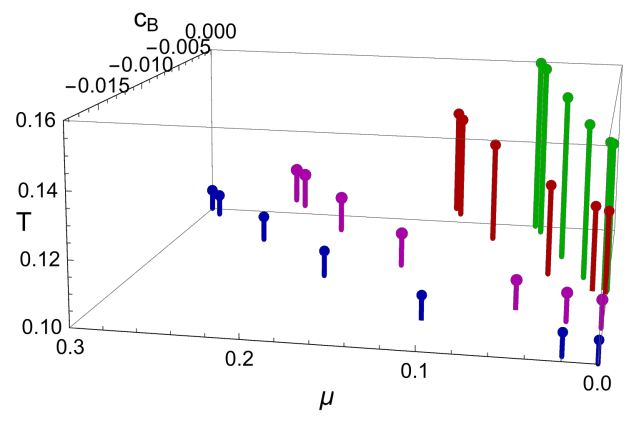

A

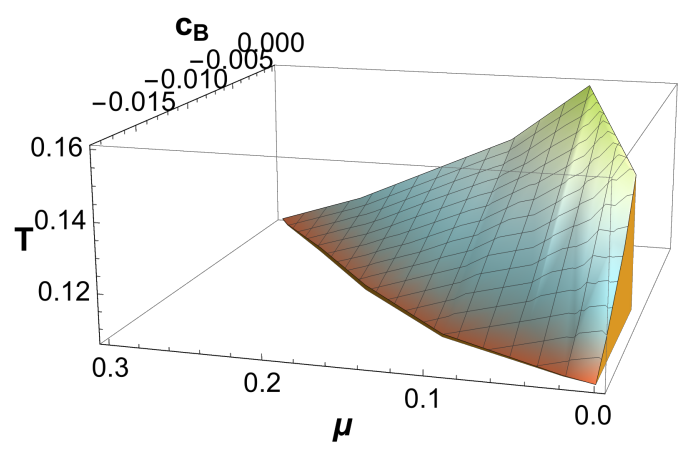

B

Figure 11. Temperature of critical end-points $\mathrm{CEP}_{H Q}$ for heavy quarks model depending on $c_{B}$ and $\mu$ for $1 \leq \nu \leq 4.5$ as a set of point (A) and as a $3 \mathrm{D}$-surface $(\mathrm{B}), c=0.227$. $T$ and $\mu$ are in units of $\mathrm{GeV}$, and $c_{B}$ of $\mathrm{GeV}^{2}$.

It is known that the deformation of the warp-factor by adding $d z^{5}$ changes IMC to MC for $d$ large enough [41]. If one considers a deformation by quadratic factor $p z^{4}$, then for particular $p$, suitable to fit Regge spectrum, one gets MC for small and medium $B$, meanwhile one observes IMC for large $B$ [42]. It is interesting to study these phenomena also for the anizotropic cases.

\subsection{Temporal Wilson loops}

To calculate the expectation value of the temporal Wilson loop

$$
W\left[C_{\vartheta}\right]=e^{-S_{\vartheta, t}},
$$

oriented along vector $\vec{n}$

$$
n_{x 1}=\cos \vartheta \sin \alpha, \quad n_{x 2}=\sin \vartheta \sin \alpha, \quad n_{x 3}=\cos \alpha,
$$

we use our metric (2.3) as a background:

$$
d s^{2}=G_{\mu \nu} d x^{\mu} d x^{\nu}=\frac{L^{2}}{z^{2}} \mathfrak{b}_{s}(z)\left[-g(z) d t^{2}+\mathfrak{g}_{1} d x^{2}+\mathfrak{g}_{2} d y_{1}^{2}+\mathfrak{g}_{3} d y_{2}^{2}+\frac{d z^{2}}{g(z)}\right]
$$




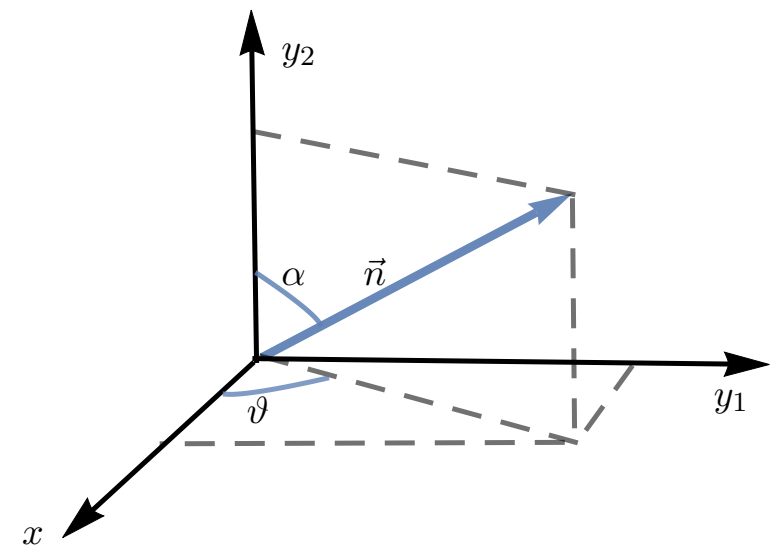

Figure 12. World sheet parametrization for the Wilson loop orientation vector $\vec{n}$.

where $\mathfrak{b}_{s}(z)=\mathfrak{b}(z) \exp (\sqrt{2 / 3} \phi(z))$ is the model warp-factor in string frame and $\mathfrak{g}_{i}$ are the corresponding $G_{\mu \nu}$-metric components. Following the holographic approach we calculate the value of the Nambu-Goto action for test string in our background in string frame:

$$
S=\frac{1}{2 \pi \alpha^{\prime}} \int d \xi^{0} d \xi^{1} \sqrt{-\operatorname{det} h_{\alpha \beta}}, \quad h_{\alpha \beta}=G_{\mu \nu} \partial_{\alpha} X^{\mu} \partial_{\beta} X^{\nu} .
$$

The world sheet is parameterized as (figure 12)

$$
\begin{aligned}
X^{0} \equiv t=\xi^{0}, & X^{1} \equiv x=\xi^{1} \cos \vartheta \sin \alpha, & & X^{2} \equiv y_{1}=\xi^{1} \sin \vartheta \sin \alpha \\
& X^{3} \equiv y_{2}=\xi^{1} \cos \alpha, & & X^{4} \equiv z=z\left(\xi^{1}\right) .
\end{aligned}
$$

Let us denote $\xi \equiv \xi^{1}$, rewrite Nambu-Goto action (3.8)

$$
\begin{aligned}
S & =-\frac{\tau}{2 \pi \alpha^{\prime}} \mathcal{S}, \quad \mathcal{S}=\int_{-\ell / 2}^{\ell / 2} d \xi M(z(\xi)) \sqrt{\mathcal{F}(z(\xi))+\left(z^{\prime}(\xi)\right)^{2}}, \\
M(z(\xi)) & =\frac{\mathfrak{b}_{s}(z(\xi))}{z^{2}(\xi)}, \quad \tau=\int d \xi^{0}, \\
\mathcal{F}(z(\xi)) & =g\left(z\left(\xi^{1}\right)\right)\left(\mathfrak{g}_{1} \cos ^{2} \vartheta \sin ^{2} \alpha+\mathfrak{g}_{2} \sin ^{2} \vartheta \sin ^{2} \alpha+\mathfrak{g}_{3} \cos ^{2} \alpha\right)
\end{aligned}
$$

and introduce the effective "potential"

$$
\begin{aligned}
\mathcal{V}(z(\xi)) & \equiv M(z(\xi)) \sqrt{\mathcal{F}(z(\xi))}= \\
& =\frac{\mathfrak{b}_{s}(z(\xi))}{\left(z^{2}(\xi)\right)} \sqrt{g(z(\xi))\left(\mathfrak{g}_{1} \cos ^{2} \vartheta \sin ^{2} \alpha+\mathfrak{g}_{2} \sin ^{2} \vartheta \sin ^{2} \alpha+\mathfrak{g}_{3} \cos ^{2} \alpha\right)}
\end{aligned}
$$

The action $\mathcal{S}$ (3.10) defines the dynamical system with a dynamic variable $z=z(\xi)$ and time $\xi$ (see for example [32]). This system has the first integral

$$
\frac{M(z(\xi)) \mathcal{F}(z(\xi))}{\sqrt{\mathcal{F}(z(\xi))+\left(z^{\prime}(\xi)\right)^{2}}}=\mathcal{I} .
$$


From (3.12) we find the "top", or the turning point, $z_{0}$ (the closed position of the minimal surface to the horizon), where $z^{\prime}(\xi)=0$ :

$$
M\left(z_{0}\right) \sqrt{F\left(z_{0}\right)}=\mathcal{I}
$$

Finding $z^{\prime}$ from (3.12) one gets representations for the string length $\ell$ and the action $\mathcal{S}(3.10)$ :

$$
\begin{aligned}
& \frac{\ell}{2}=\int_{0}^{z_{0}} \frac{1}{\sqrt{\mathcal{F}(z)}} \frac{d z}{\sqrt{\frac{\mathcal{V}^{2}(z)}{\mathcal{V}^{2}\left(z_{0}\right)}-1}} \\
& \frac{\mathcal{S}}{2}=\int_{\epsilon}^{z_{0}} \frac{M(z) d z}{\sqrt{1-\frac{\mathcal{V}^{2}\left(z_{0}\right)}{\mathcal{V}^{2}(z)}}}
\end{aligned}
$$

To obtain the string tension we have to calculate asymptotic of $\mathcal{S}$ for $\ell \rightarrow \infty$. If the stationary point of $\mathcal{V}(z)$ exists in the region $0<z<z_{h}$,

$$
\left.\mathcal{V}^{\prime}\right|_{z_{D W}}=0
$$

we call this point a dynamical wall (DW) point and take the top point $z_{0}$ equal to the DW position, $z_{0}=z_{D W}$. Near this point we get

$$
\begin{aligned}
& \ell \underset{z \rightarrow z_{D W}}{\sim} \frac{1}{\sqrt{F\left(z_{D W}\right)}} \sqrt{\frac{\mathcal{V}\left(z_{D W}\right)}{\mathcal{V}^{\prime \prime}\left(z_{D W}\right)}} \log \left(z-z_{D W}\right) \underset{z \rightarrow z_{D W}}{\rightarrow} \infty, \\
& \mathcal{S} \underset{z \rightarrow z_{D W}}{\sim} M\left(z_{D W}\right) \sqrt{\frac{\mathcal{V}\left(z_{D W}\right)}{\mathcal{V}^{\prime \prime}\left(z_{D W}\right)}} \log \left(z-z_{D W}\right) .
\end{aligned}
$$

Hence for the string tension we get

$$
\mathcal{S} \sim \sigma_{D W} \cdot \ell, \quad \sigma_{D W}=M\left(z_{D W}\right) \sqrt{F\left(z_{D W}\right)} .
$$

If there is no stationary point of $\mathcal{V}(z)$ in the region $0<z<z_{h}$, the string tension becomes

$$
\sigma_{h}=M\left(z_{h}\right) \sqrt{F\left(z_{h}\right)}
$$

Thus the turning points for Wilson loops $W L x, W L y_{1}$ and $W L y_{2}$, oriented along $x$, $y_{1}$ and $y_{2}$ axes, respectively, are defined by equations:

$$
\begin{array}{ll}
W L x: & -c z+\sqrt{\frac{2}{3}} \phi^{\prime}(z)+\frac{g^{\prime}}{2 g}-\left.\frac{2}{z}\right|_{z=z_{D W x}} ^{=0} \\
W L_{y_{1}}: & -c z+\sqrt{\frac{2}{3}} \phi^{\prime}(z)+\frac{g^{\prime}}{2 g}-\left.\frac{\nu+1}{\nu z}\right|_{z=z_{D W y_{1}}} ^{=0,} \\
W L_{y_{2}}: & -c z+\sqrt{\frac{2}{3}} \phi^{\prime}(z)+\frac{g^{\prime}}{2 g}-\frac{\nu+1}{\nu z}+\left.c_{B} z\right|_{z=z_{D W y_{2}}} ^{=0 .}
\end{array}
$$

It is obvious, that for $c_{B}=0$ expressions in (3.22) and (3.23) become equal, and putting $\nu=1$ reduces all equations $(3.21)-(3.23)$ to $(3.21)$. The lines in the plane $\left(\mu, z_{h}\right)$ and 


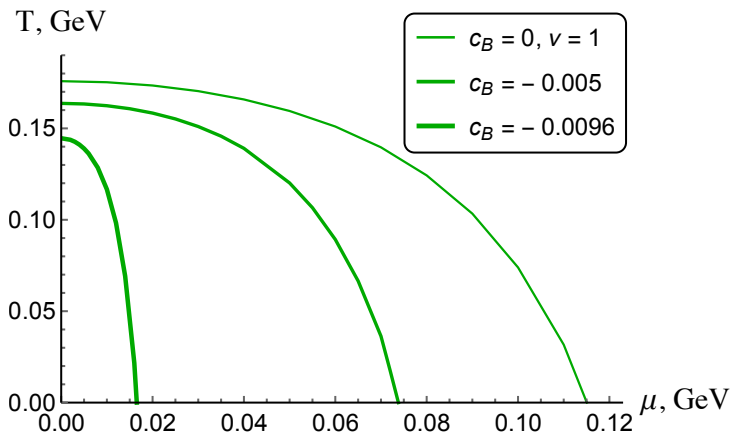

A

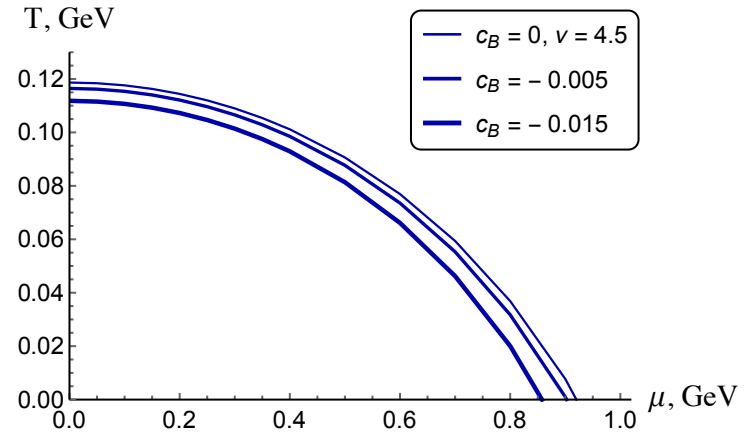

$\mathrm{B}$

Figure 13. Wilson loop transition lines for $W L y_{2}$, different $c_{B}$ and for $\nu=1(\mathrm{~A})$ and $\nu=4.5(\mathrm{~B})$; $c=0.227$.

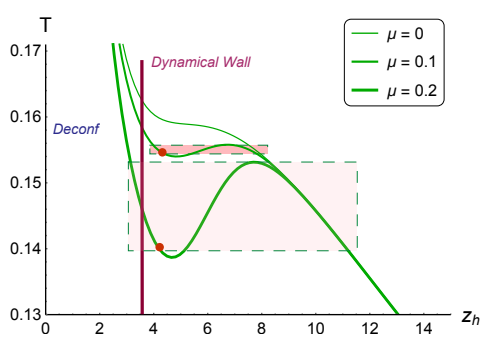

A

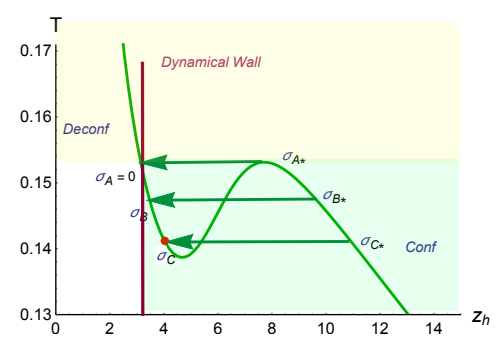

$\mathrm{B}$

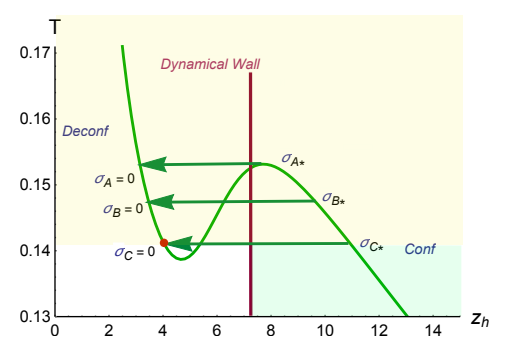

$\mathrm{C}$

Figure 14. Scheme of confinement/deconfuinement depending on location of the BH-BH phase transition points relative to the dynamic wall. $T$ is in units of $\mathrm{GeV}$ and $z_{h}$ of $\mathrm{GeV}^{-1}$.

therefore in the plane $(\mu, T)$, where equations (3.21)-(3.23) stop having real solutions, are interpreted as lines of Wilson phase transition. Locations in the $(\mu, T)$-plane of phase transition lines for Wilson loops $W L x$ and $W L y_{1}$ were already considered [30, 32].

On figure 13 phase transition lines for Wilson loop $W L y_{2}$ are presented. The case $c_{B}=$ 0 obviously corresponds also to $W L y_{1}$ transition curves. We see, that the appearance of external magnetic field $\left(c_{B} \neq 0\right)$ makes the Wilson loop phase transition line to shrink. For $\nu=1$ maximum chemical potential at $T=0$ decreases faster than maximum temperature at $\mu=0$. For $\nu=4.5$ the shrinkage of the phase transition line corresponding to Wilson loop $W L y_{2}$ is much weaker and looks almost cocentric.

In the backgrounds with instability one has also to check that the extended surface lies in the stability zone [53]. This allows to determine roles of different transition lines in the general confinement/deconfinement picture.

There are different situations in this matter. For given $\mu$ the dynamic wall can be out of instability zone (small rectangular pink region on figure 14 A), or cross the instability zone starting from some temperature (large rectangular pink region on figure $14 \mathrm{~A}$ ). In the first case the jump to the stable zone keeps confinement. The detailed analysis shows, that the string tension decreases from $\sigma_{*}=\sigma\left(z_{D W^{*}}\right)$ in unstable phase to $\sigma=\sigma\left(z_{D W}\right)$ in the stable phase. Here $z_{D W}$ is calculated for $z_{h}$, and $z_{D W^{*}}$ is calculated for $z_{h}^{*}$, such that 

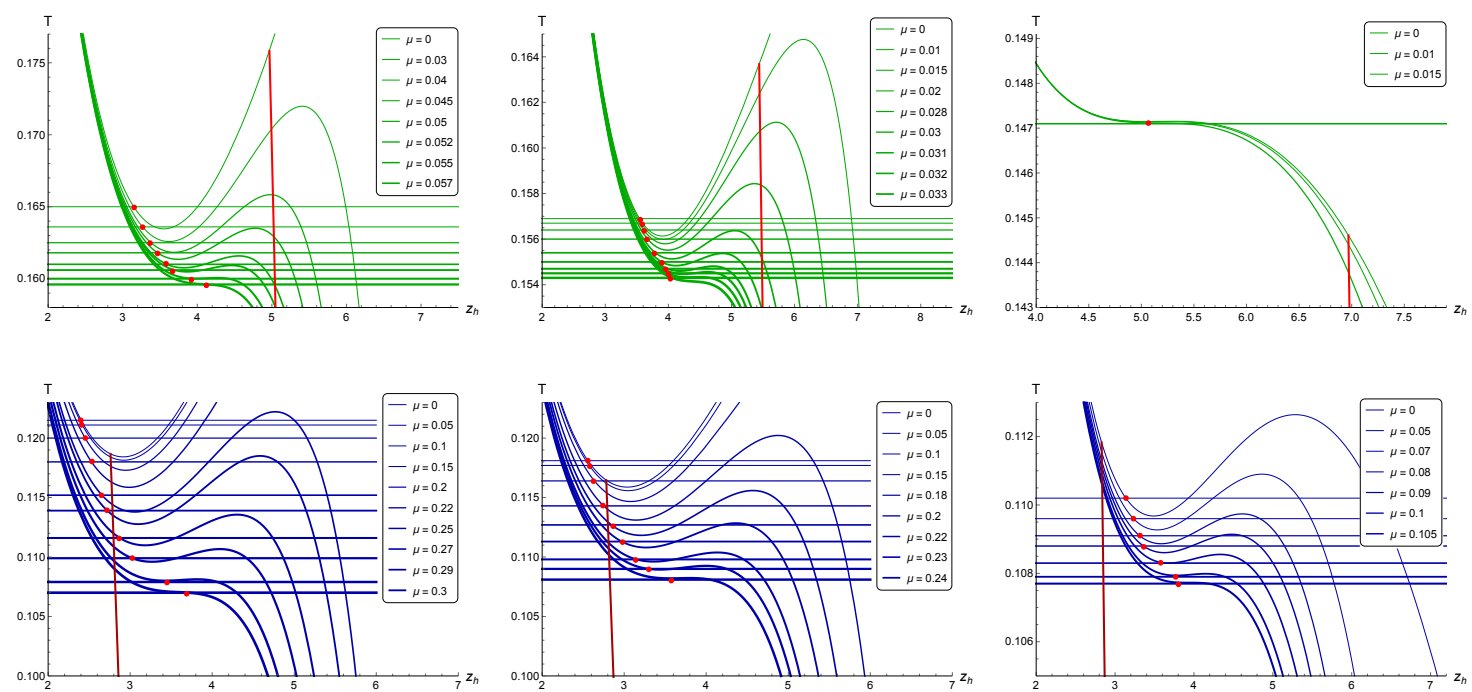

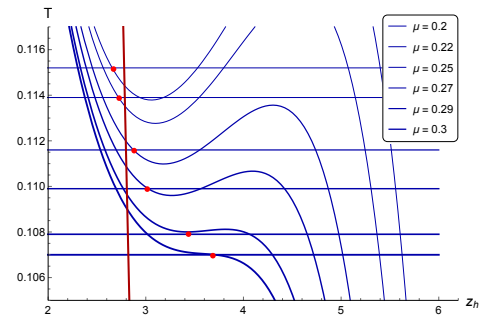

$\mathrm{A}$

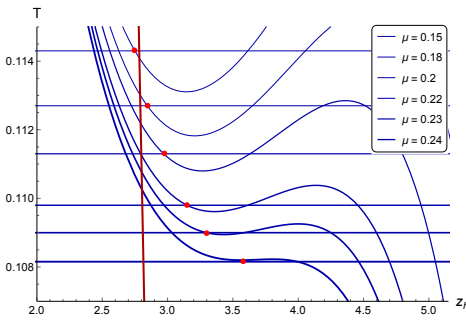

$\mathrm{B}$

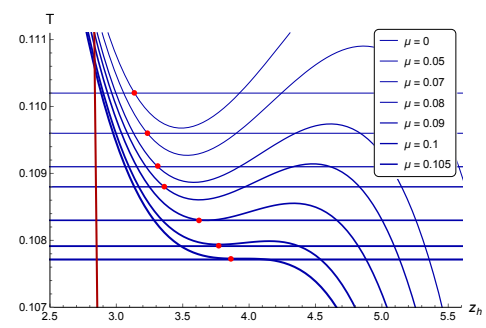

$\mathrm{C}$

Figure 15. Location of the BH-BH phase transition points (red point) relative to the dynamic wall (red lines) for $\nu=1$ (first line) and $\nu=4.5$ (second and third line, third line is the zoom of the second) for $c_{B}=0(\mathrm{~A}), c_{B}=-0.005(\mathrm{~B}), c_{B}=-0.0096$ for $\nu=1$ and $c_{B}=-0.015$ for $\nu=4.5$, $c=0.227$. $T$ is in units of $\mathrm{GeV}$ and $z_{h}$ of $\mathrm{GeV}^{-1}$.

$T\left(z_{h}^{*}, \mu\right)=T\left(z_{h}, \mu\right)$. It is tempting to interpret the phase, where dynamical walls exist for $z_{h}$ and $z_{h}^{*}$, as quarkyonic phase (figure $14 \mathrm{~B}$ ). If the DW lies in unstable zone, we lose the linear interaction between quarks in the quarkyonic phase (figure $14 \mathrm{C}$ ).

This is exactly the situation that takes place for $\nu=1$ in our model (first line in figure 15). The dynamic wall is located in the instability region, and the $\mathrm{BH}-\mathrm{BH}$ phase transition make us land in the area of deconfinement (figure $15 \mathrm{~A}, \mathrm{~B}$, first line). So in the absence of the primary anisotropy confinement/deconfinement phase transition is detemined by the first order phase transition (BH-BH line) till the intersection with the WL-line, providing us with the crossover. The part of the $\mathrm{BH}-\mathrm{BH}$ line, that turns out to be in the deconfinement area to the right of the crossover (figure $16 \mathrm{~A}, \mathrm{C}$ ), seems to cause density jump in the QGP. For large $c_{B} \mathrm{BH}-\mathrm{BH}$ phase transition lies above the $\mathrm{DW}$, that is still in the instability region (figure $15 \mathrm{C}$, first line). So the entire $\mathrm{BH}-\mathrm{BH}$ line serves for density jumps in QGP, not separating confinement and deconfinement areas (figure 16 E).

For $\nu=4.5$ the situation is quite different, as the dynamic wall is located in the stable region. In the absence of external magnetic field first order phase transition lands into the deconfinement region for $0 \leq \mu \leq 0.2448$, closing the crossover from above till the 
intersection with the WL-line. For $\mu>0.2448$ the Hawling-Page-like phase transition lands in the confinement area (figure $15 \mathrm{~A}$, second line). This time the $\mathrm{BH}-\mathrm{BH}$ line produces the density jump of the confined matter, while confinement/deconfinement phase transition is determined by the $y$-Wilson loop. (Let us to remind that for $c_{B}=0$ there is no difference between $y_{1}$ and $y_{2}$ orientations.) On figure $15 \mathrm{~A}$ in the third line show the same picture more clearly, zooming the $\mathrm{BH}-\mathrm{BH}$ transition to the confinement area from figure $15 \mathrm{~A}$, second line.

Adding the external magnetic field makes BH-BH line go under the WL-line into the confinement area earlier, i.e. for lesser $\mu$ values (figure $15 \mathrm{~B}$, second line and zoom in figure $15 \mathrm{~B}$, third line). The curved triangle limited by the intercection point from the left, CEP temperature from the right, BH-BH line from below and WL from above should represent the area of higher density in the confinement area (figure $16 \mathrm{~B}, \mathrm{D}$ ).

When the coupling coefficient reaches critical value $c_{B \text { crit }} \approx-0.11$ the entire BHBH line sinks into the confinement area. Therefore the confinement/deconfinement phase transition goes along the Wilson loop line only (figure $15 \mathrm{C}$, second line and zoom in figure $15 \mathrm{C}$, third line), and we have a higher density sector between the $\mathrm{BH}-\mathrm{BH}$ and WLlines (figure $16 \mathrm{~F}$ ).

On figure 16 joint phase transition diagrams for $\mathrm{BH}-\mathrm{BH}$ phase transition lines and $y_{2}$-oriented Wilson loops are displayed. We see the picture quite similar to our previous results for heavy quarks.

For $\nu=1$ without magnetic field (figure $16 \mathrm{~A}$ ) BH-BH line determines the confinement/deconfinement phase transition for $0 \leq \mu \leq \mu_{b y_{2}}=0.04666$ till the intersection with the $y_{2}$ Wilson loop line at point $(0.04666 ; 0.1617)$.

If we turn on the external magnetic field, the intersection between $\mathrm{BH}-\mathrm{BH}$ line and Wilson loop line shifts to smaller temperature and chemical potential, $(0.0235 ; 0.1558)$ for $c_{B}=-0.005$ (figure $16 \mathrm{C}$ ), and the total area of confinement phase predictably decreases. Also phase transition lines, corresponding to the background (BH-BH) and string action $\left(\mathrm{WLy}_{2}\right)$, become closer to each other.

When the coupling with the $F_{\mu \nu}^{(B)}$ is almost maximum $\left(c_{B}=-0.0096\right)$, BH-BH transition line turns out to be above the $y_{2}$ Wilson loop line without any intersection (figure $16 \mathrm{E}$ ).

For $\nu=4.5$ the picture can be called the opposite from some point of view. Without the external magnetic field (figure 16 B) $y_{2}$ Wilson loop line plays the main role for $\mu \leq$ $\mu_{y_{2} b}=0.2448$ till the intersection with the $\mathrm{BH}-\mathrm{BH}$ line at $(0.2448 ; 0.1121)$. BH-BH phase transition line lasts till the $\mathrm{CEP}_{H Q}$ at $(0.3 ; 0.107)$, where the main role comes back to Wilson loop line via the jump to higher temperature.

For $c_{B}=-0.005$ the picture remains the same qualitatively (figure $16 \mathrm{D}$ ). Transition lines intersect at point $(0.1753 ; 0.113)$, i.e. at lesser chemical potential, but a little higher temperature.

Eventually $\mathrm{BH}-\mathrm{BH}$ transition line turns out to lie entirely under the WL-line till $(0.24,0.1081)$, where it's CEP is located (figure $16 \mathrm{~F})$. Critical value of the coupling function, for which $\left.T_{B H-B H}\right|_{\mu=0}=\left.T_{W L y_{2}}\right|_{\mu=0}$ is about $c_{B \text { crit }} \approx-0.11$ in this case. 
$\mathrm{T}, \mathrm{GeV}$

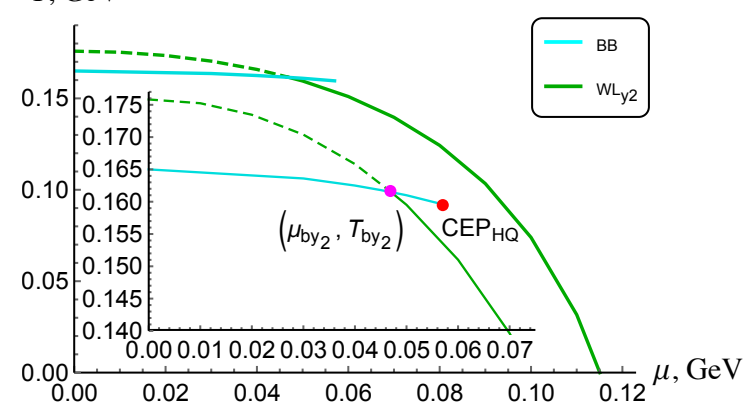

A

$\mathrm{T}, \mathrm{GeV}$

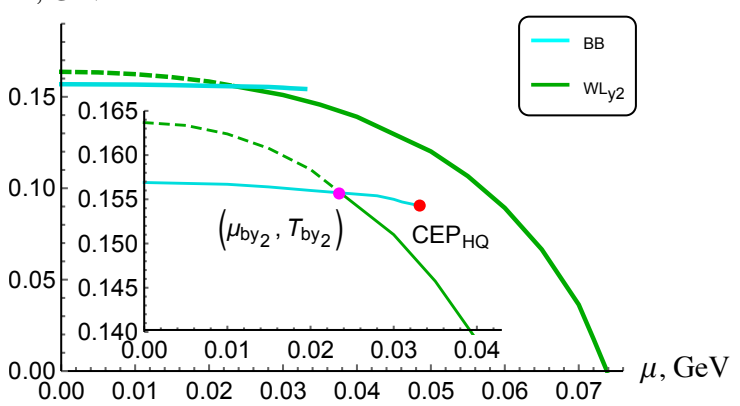

$\mathrm{C}$

$\mathrm{T}, \mathrm{GeV}$

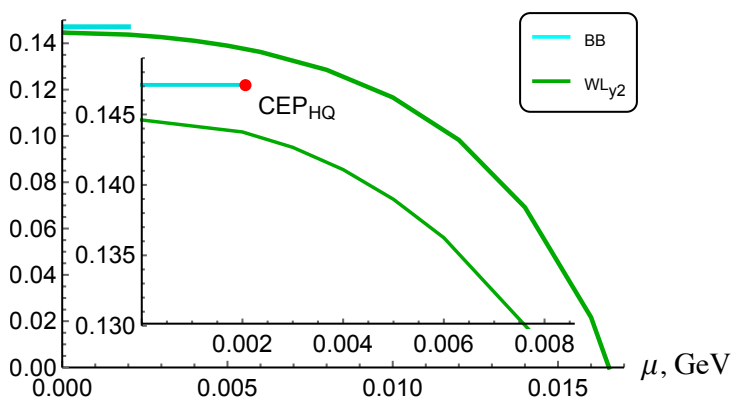

$\mathrm{E}$

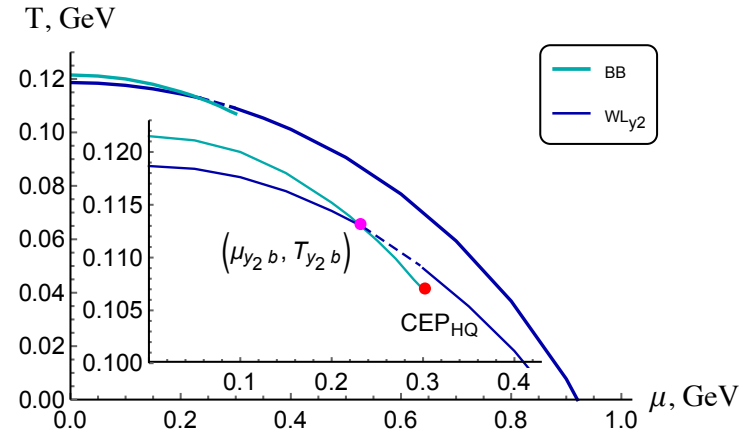

B

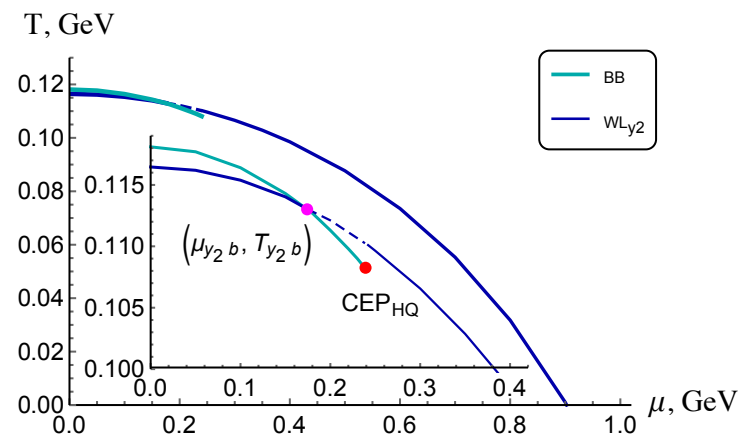

$\mathrm{D}$

\section{$\mathrm{T}, \mathrm{GeV}$}

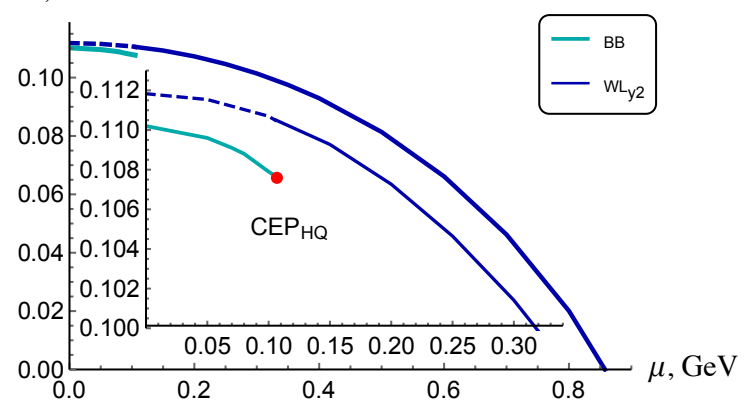

F

Figure 16. Phase diagram for $y_{2}$-orientation for $c_{B}=0, \nu=1(\mathrm{~A})$ and $\nu=4.5(\mathrm{~B}), c_{B}=-0.005$, $\nu=1(\mathrm{C})$ and $\nu=4.5(\mathrm{D}), c_{B}=-0.0096, \nu=1(\mathrm{E})$ and $c_{B}=-0.015, \nu=4.5(\mathrm{~F}) ; c=0.227$.

\section{Conclusions}

In this work holographic description of hot dense anisotropic QGP in magnetic field for heavy quarks model was constructed. This model is the extension of the previous solution [30] that serves as a zero magnetic field limit.

The magnetic field $F^{(B)}$ is characterized by two parameters - "charge" $F_{x y_{1}}^{(B)}=q_{B}$ and metric coupling coefficient $c_{B}$. First of them characterises the Maxwell field itself, while the second one describes the metric deformation due to the presence of external magnetic field. In our model kinetic coupling function has simplest gaussian form [27, 36, 42] and depends of the coefficient $c_{B}$ and the external "charge" $q_{B}$ which not connected to each other. Such 


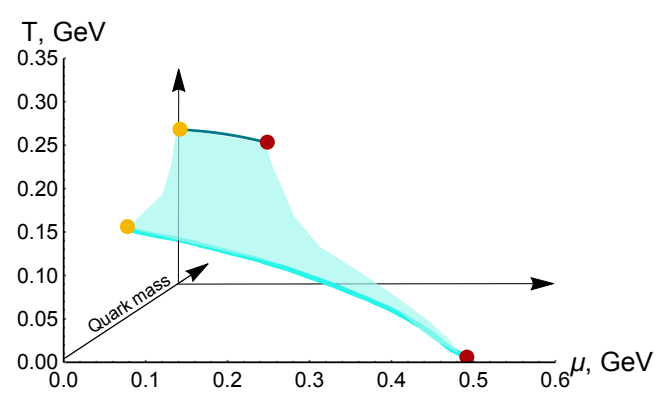

A

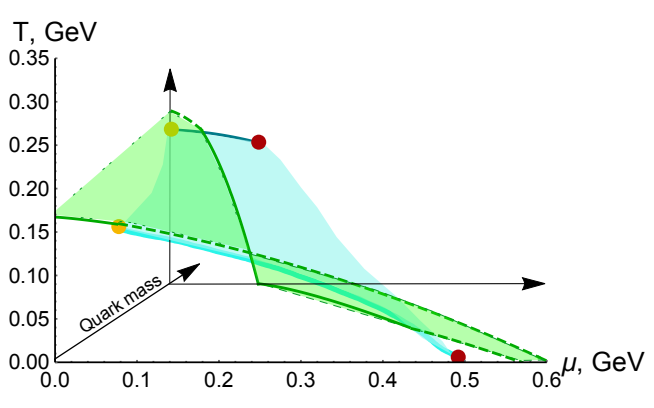

$\mathrm{B}$

Figure 17. Schematic pictures of phase transitions dependence on quark mass. The cyan surface shows the first order phase transition (A) and green surface shows the confinement/deconfinement transition surface (A, B). The red and yellow points are critical end points.

a parameterization was introduced for more generality of the model. As a result, it turns out that the magnetic "charge" is almost not involved into the main properties of our holographic description for QGP, so we actually consider $c_{B}$ as an efficient factor of the Maxwell field $F_{x y_{1}}^{(B)}$ and put $q_{B}=1$. But this doesn't mean at all that the phase transition structure is independent from the strength of external magnetic field. The fact is that we can always link these parameters determining coupling coefficient as a function of "charge", $c_{B}=f\left(q_{B}\right)$. The specific shape of this function is an important aspect that should allow to fit lattice results, experimental data as well as reach agreement with other theoretical considerations.

The structure of phase transitions strongly depends on the chosen model - models corresponding to light/heavy quarks have different structures. In fact there is finer division depending on quark type taken into account, see for example [78-80]. In the holographic approach light/heavy quarks models have different structures of the background phase transition [16, 28]. In most of holographic models the background phase transition, see figure $17 \mathrm{~A}$, is related to chiral symmetry breaking [15, 22, 24, 33, 34, 40, 41, 44, 48, 49, 51]. Confinement/deconfinement phase transition may be related to background phase transition and may be not, figure $17 \mathrm{~B}$. This depends on the relative position of the dynamic wall and the background phase transition, see more detail discussion in section 3.3. An expected phase structure for the intermediate version of the model between heavy and light quarks (the so-called "hybrid" model) is shown on figure $17 \mathrm{~A}$ and figure $17 \mathrm{~B}$. The area of the assumed location of the first-order phase transition line is depicted in cyan.

In this paper we investigated the dependence of the phase transition structure on the anisotropy, characterized by parameter $\nu$ (see eq. (2.3)), and magnetic fields. Our results are schematically shown in the $3 \mathrm{D}$ diagrams $(\mu, \nu, T)$ and $(\mu, B, T)$ on figure 18 . The WL phase transition is indicated by green lines, the $\mathrm{BH}-\mathrm{BH}$ phase transition is indicated by cyan, blue and magenta lines. The solid lines of BH-BH and WL phase transitions indicate the confinement/deconfinement phase transition. In the figure $18 \mathrm{~A}$ we see that while increasing anisotropy, the BH-BH phase transition and WL phase transition occur at lower temperatures for the same values of the chemical potential. With the increase of $\nu$, the 


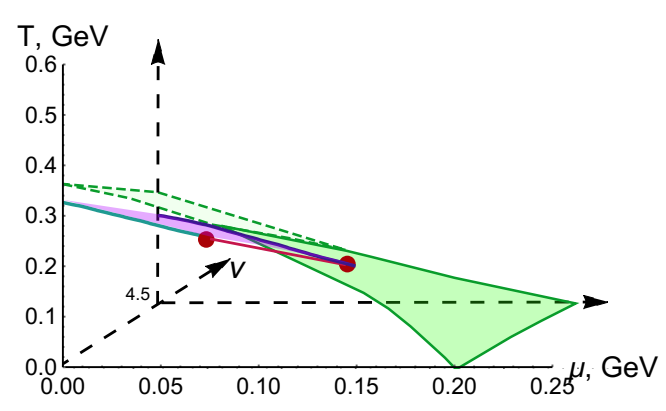

A

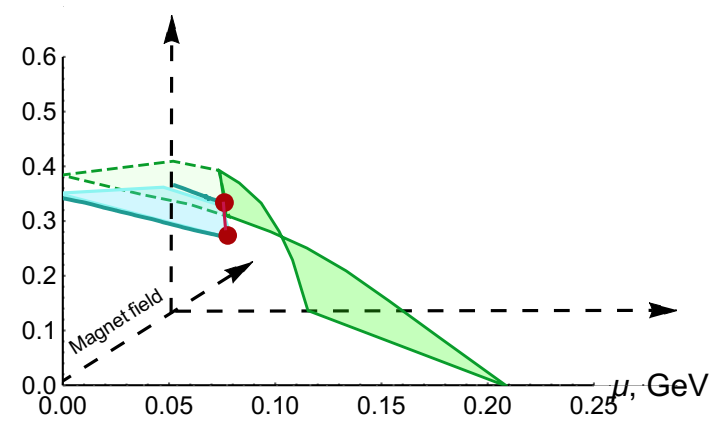

B

Figure 18. Schematic representations of the structure of phase transitions of a model describing heavy quarks depending on the anisotropy caused by: parameter $\nu(\mathrm{A})$, magnetic field (B).

first-order phase transition line elongates. In the figure $18 \mathrm{~B}$, we see qualitatively the same situation with increasing magnetic field, the phase transition occurs at lower temperature values for the same chemical potential values, but the first-order phase transition line shortens.

To complete the confinement/deconfinement picture we also investigated string properties of the solution - string tension depending of orientation of the quark pair. For this purpose we investigated behavior of differently oriented temporal Wilson loops, that determines location of confinement/deconfinement phase transition. Note that unlike the dynamic walls' positions the string tension strongly depends on the integration boundary for the scalar field [47]. Here we performed calculations for the simplest case $z_{0}=\epsilon \ll 1$ only. Choice of the function $z_{0}=f\left(z_{h}\right)$ will be consider in further investigations of the model.

In the model considered in this paper we have observed only IMC. Such a behavior distinguishes this model from more complicated heavy quarks models with higher order polynomials in the warp-factor. Namely, the model considered in [42] demonstrates MC for small magnetic fields and IMC for large ones. This phenomena takes place for small values of the chemical potential, while for large admissible chemical potentials the model exhibits MC only. Similar to the simplest heavy quarks models [21, 30] the model has CEPs, those position depend on values of magnetic field. It seems, that this phenomena will take place also for the case of the warp factor considered in [36].

Note, that to get more realistic description of the phase structure we have to deal with so-called hybrid warped factor that is a combination of the exponent of polynomials with logarithmic corrections. Constructing of such a superposition model is a must-have item on the list of future research.

\section{Acknowledgments}

This work is supported by Russian Science Foundation grant No. 20-12-00200.

Open Access. This article is distributed under the terms of the Creative Commons Attribution License (CC-BY 4.0), which permits any use, distribution and reproduction in any medium, provided the original author(s) and source are credited. 


\section{References}

[1] J. Casalderrey-Solana, H. Liu, D. Mateos, K. Rajagopal and U.A. Wiedemann, Gauge/String Duality, Hot QCD and Heavy Ion Collisions, Cambridge University Press, Cambridge U.K. (2014) [arXiv:1101.0618] [INSPIRE].

[2] I. Ya. Aref'eva, Holographic approach to quark-gluon plasma in heavy ion collisions, Phys. Usp. 57 (2014) 527.

[3] O. DeWolfe, S.S. Gubser, C. Rosen and D. Teaney, Heavy ions and string theory, Prog. Part. Nucl. Phys. 75 (2014) 86 [arXiv: 1304.7794] [InSPIRE].

[4] E. Witten, Anti-de Sitter space, thermal phase transition, and confinement in gauge theories, Adv. Theor. Math. Phys. 2 (1998) 505 [hep-th/9803131] [InSPIRE].

[5] T. Sakai and S. Sugimoto, Low energy hadron physics in holographic QCD, Prog. Theor. Phys. 113 (2005) 843 [hep-th/0412141] [INSPIRE].

[6] O. Andreev and V.I. Zakharov, On Heavy-Quark Free Energies, Entropies, Polyakov Loop, and $A d S / Q C D, J H E P 04$ (2007) 100 [hep-ph/0611304] [INSPIRE].

[7] U. Gürsoy, E. Kiritsis, L. Mazzanti and F. Nitti, Holography and Thermodynamics of 5D Dilaton-gravity, JHEP 05 (2009) 033 [arXiv:0812.0792] [INSPIRE].

[8] U. Gürsoy, E. Kiritsis, L. Mazzanti and F. Nitti, Improved Holographic Yang-Mills at Finite Temperature: Comparison with Data, Nucl. Phys. B 820 (2009) 148 [arXiv:0903.2859] [INSPIRE].

[9] S. He, M. Huang and Q.-S. Yan, Logarithmic correction in the deformed $\mathrm{AdS}_{5}$ model to produce the heavy quark potential and QCD $\beta$-function, Phys. Rev. D 83 (2011) 045034 [arXiv:1004.1880] [INSPIRE].

[10] M. Mia, K. Dasgupta, C. Gale and S. Jeon, Heavy Quarkonium Melting in Large N Thermal QCD, Phys. Lett. B 694 (2011) 460 [arXiv:1006.0055] [INSPIRE].

[11] U. Gürsoy, E. Kiritsis, L. Mazzanti, G. Michalogiorgakis and F. Nitti, Improved Holographic QCD, Lect. Notes Phys. 828 (2011) 79 [arXiv:1006.5461] [INSPIRE].

[12] P. Colangelo, F. Giannuzzi, S. Nicotri and V. Tangorra, Temperature and quark density effects on the chiral condensate: An AdS/QCD study, Eur. Phys. J. C $\mathbf{7 2}$ (2012) 2096 [arXiv: 1112.4402] [INSPIRE].

[13] R.-G. Cai, S. He and D. Li, A hQCD model and its phase diagram in Einstein-Maxwell-Dilaton system, JHEP 03 (2012) 033 [arXiv:1201.0820] [INSPIRE].

[14] D. Giataganas, Probing strongly coupled anisotropic plasma, JHEP 07 (2012) 031 [arXiv: 1202.4436] [INSPIRE].

[15] D. Li, M. Huang and Q.-S. Yan, A dynamical soft-wall holographic QCD model for chiral symmetry breaking and linear confinement, Eur. Phys. J. C 73 (2013) 2615 [arXiv: 1206.2824] [INSPIRE].

[16] S. He, S.-Y. Wu, Y. Yang and P.-H. Yuan, Phase Structure in a Dynamical Soft-Wall Holographic QCD Model, JHEP 04 (2013) 093 [arXiv: 1301.0385] [INSPIRE].

[17] D. Li and M. Huang, Dynamical holographic QCD model for glueball and light meson spectra, JHEP 11 (2013) 088 [arXiv: 1303.6929] [INSPIRE]. 
[18] Y. Yang and P.-H. Yuan, A Refined Holographic QCD Model and QCD Phase Structure, JHEP 11 (2014) 149 [arXiv: 1406.1865] [INSPIRE].

[19] D. Li, S. He and M. Huang, Temperature dependent transport coefficients in a dynamical holographic QCD model, JHEP 06 (2015) 046 [arXiv:1411.5332] [INSPIRE].

[20] R. Rougemont, R. Critelli and J. Noronha, Holographic calculation of the QCD crossover temperature in a magnetic field, Phys. Rev. D 93 (2016) 045013 [arXiv: 1505.07894] [INSPIRE].

[21] Y. Yang and P.-H. Yuan, Confinement-deconfinement phase transition for heavy quarks in a soft wall holographic QCD model, JHEP 12 (2015) 161 [arXiv:1506. 05930] [INSPIRE].

[22] K. Chelabi, Z. Fang, M. Huang, D. Li and Y.-L. Wu, Realization of chiral symmetry breaking and restoration in holographic QCD, Phys. Rev. D 93 (2016) 101901 [arXiv:1511.02721] [INSPIRE].

[23] Z. Fang, S. He and D. Li, Chiral and Deconfining Phase Transitions from Holographic QCD Study, Nucl. Phys. B 907 (2016) 187 [arXiv:1512.04062] [inSPIRE].

[24] K. Chelabi, Z. Fang, M. Huang, D. Li and Y.-L. Wu, Chiral Phase Transition in the Soft-Wall Model of AdS/QCD, JHEP 04 (2016) 036 [arXiv:1512.06493] [INSPIRE].

[25] D. Li, M. Huang, Y. Yang and P.-H. Yuan, Inverse Magnetic Catalysis in the Soft-Wall Model of AdS/QCD, JHEP 02 (2017) 030 [arXiv:1610.04618] [INSPIRE].

[26] D. Li and M. Huang, Chiral phase transition of QCD with $N_{f}=2+1$ flavors from holography, JHEP 02 (2017) 042 [arXiv: 1610.09814] [INSPIRE].

[27] D. Dudal and S. Mahapatra, Confining gauge theories and holographic entanglement entropy with a magnetic field, JHEP 04 (2017) 031 [arXiv: 1612.06248] [INSPIRE].

[28] M.-W. Li, Y. Yang and P.-H. Yuan, Approaching Confinement Structure for Light Quarks in a Holographic Soft Wall QCD Model, Phys. Rev. D 96 (2017) 066013 [arXiv:1703.09184] [INSPIRE].

[29] Y. Yang and P.-H. Yuan, Universal Behaviors of Speed of Sound from Holography, Phys. Rev. D 97 (2018) 126009 [arXiv: 1705.07587] [InSPIRE].

[30] I. Aref'eva and K. Rannu, Holographic Anisotropic Background with Confinement-Deconfinement Phase Transition, JHEP 05 (2018) 206 [arXiv:1802.05652] [INSPIRE].

[31] I.Y. Aref'eva, A.A. Golubtsova and G. Policastro, Exact holographic RG flows and the $A_{1} \times A_{1}$ Toda chain, JHEP 05 (2019) 117 [arXiv: 1803.06764] [INSPIRE].

[32] I. Aref'eva, K. Rannu and P. Slepov, Orientation Dependence of Confinement-Deconfinement Phase Transition in Anisotropic Media, Phys. Lett. B 792 (2019) 470 [arXiv:1808.05596] [INSPIRE].

[33] Z. Fang, Y.-L. Wu and L. Zhang, Chiral phase transition and QCD phase diagram from AdS/QCD, Phys. Rev. D 99 (2019) 034028 [arXiv:1810.12525] [INSPIRE].

[34] J. Chen, S. He, M. Huang and D. Li, Critical exponents of finite temperature chiral phase transition in soft-wall AdS/QCD models, JHEP 01 (2019) 165 [arXiv:1810.07019] [INSPIRE].

[35] A.A. Golubtsova and V.H. Nguyen, Wilson Loops in Exact Holographic RG Flows at Zero and Finite Temperatures, Theor. Math. Phys. 202 (2020) 214 [arXiv:1906.12316] [InSPIRE]. 
[36] H. Bohra, D. Dudal, A. Hajilou and S. Mahapatra, Anisotropic string tensions and inversely magnetic catalyzed deconfinement from a dynamical AdS/QCD model, Phys. Lett. B 801 (2020) 135184 [arXiv: 1907.01852] [INSPIRE].

[37] X. Chen, D. Li, D. Hou and M. Huang, Quarkyonic phase from quenched dynamical holographic QCD model, JHEP 03 (2020) 073 [arXiv:1908.02000] [INSPIRE].

[38] I. Aref'eva, K. Rannu and P. Slepov, Cornell potential for anisotropic QGP with non-zero chemical potential, EPJ Web Conf. 222 (2019) 03023 [INSPIRE].

[39] Z. Fang and Y.-L. Wu, Equation of state and chiral transition in soft-wall AdS/QCD with more realistic gravitational background, arXiv:1909.06917 [INSPIRE].

[40] Z. Fang and L. Zhang, Chiral transition and meson melting with finite chemical potential in an improved soft-wall AdS/QCD Model, arXiv:1910.02269 [INSPIRE].

[41] A. Ballon-Bayona and L.A.H. Mamani, Nonlinear realization of chiral symmetry breaking in holographic soft wall models, Phys. Rev. D 102 (2020) 026013 [arXiv: 2002.00075] [INSPIRE].

[42] S. He, Y. Yang and P.-H. Yuan, Analytic Study of Magnetic Catalysis in Holographic QCD, arXiv: 2004.01965 [INSPIRE].

[43] D. Dudal, A. Hajilou and S. Mahapatra, A quenched 2-flavour Einstein-Maxwell-dilaton gauge-gravity model, Eur. Phys. J. A 57 (2021) 142 [arXiv:2103.01185] [INSPIRE].

[44] A. Ballon-Bayona, J.P. Shock and D. Zoakos, Magnetic catalysis and the chiral condensate in holographic QCD, JHEP 10 (2020) 193 [arXiv:2005.00500] [INSPIRE].

[45] A. Ballon-Bayona, H. Boschi-Filho, E.F. Capossoli and D.M. Rodrigues, Criticality from Einstein-Maxwell-dilaton holography at finite temperature and density, Phys. Rev. D 102 (2020) 126003 [arXiv: 2006.08810] [INSPIRE].

[46] P. Colangelo, F. De Fazio and N. Losacco, Chaos in a $Q \bar{Q}$ system at finite temperature and baryon density, Phys. Rev. D 102 (2020) 074016 [arXiv:2007.06980] [INSPIRE].

[47] I.Y. Aref'eva, K. Rannu and P. Slepov, Holographic anisotropic model for light quarks with confinement-deconfinement phase transition, JHEP 06 (2021) 090 [arXiv: 2009.05562] [INSPIRE].

[48] M.-W. Li, Y. Yang and P.-H. Yuan, Analytic Study on Chiral Phase Transition in Holographic QCD, JHEP 02 (2021) 055 [arXiv:2009.05694] [INSPIRE].

[49] H. Bohra, D. Dudal, A. Hajilou and S. Mahapatra, Chiral transition in the probe approximation from an Einstein-Maxwell-dilaton gravity model, Phys. Rev. D 103 (2021) 086021 [arXiv: 2010.04578] [INSPIRE].

[50] I. Aref'eva, Holography for Nonperturbative Study of QFT, Phys. Part. Nucl. 51 (2020) 489 [INSPIRE].

[51] D.M. Rodrigues, D. Li, E. Folco Capossoli and H. Boschi-Filho, Finite density effects on chiral symmetry breaking in a magnetic field in $2+1$ dimensions from holography, Phys. Rev. D 103 (2021) 066022 [arXiv: 2010.06762] [INSPIRE].

[52] N. Jokela and J.G. Subils, Is entanglement a probe of confinement?, JHEP 02 (2021) 147 [arXiv: 2010.09392] [INSPIRE].

[53] I. Ya. Aref'eva, Theoretical studies of the formation and properties of quark-gluon matter under conditions of high baryon densities attainable at the NICA experimental complex, submitted to Phys. Part. Nucl.. 
[54] P. Slepov, A way to improve string tension dependence on temperature in holographic mode, submitted to Phys. Part. Nucl..

[55] K. Rannu, Holographic Anisotropic Model for Light Quarks with Confinement-Deconfinement Phase Transition, submitted to Phys. Part. Nucl.

[56] D. Mateos and D. Trancanelli, The anisotropic $N=4$ super Yang-Mills plasma and its instabilities, Phys. Rev. Lett. 107 (2011) 101601 [arXiv:1105.3472] [INSPIRE].

[57] D. Mateos and D. Trancanelli, Thermodynamics and Instabilities of a Strongly Coupled Anisotropic Plasma, JHEP 07 (2011) 054 [arXiv: 1106.1637] [INSPIRE].

[58] R.A. Janik and P. Witaszczyk, Towards the description of anisotropic plasma at strong coupling, JHEP 09 (2008) 026 [arXiv:0806.2141] [INSPIRE].

[59] A. Rebhan and D. Steineder, Probing Two Holographic Models of Strongly Coupled Anisotropic Plasma, JHEP 08 (2012) 020 [arXiv: 1205.4684] [INSPIRE].

[60] I.Y. Aref'eva and A.A. Golubtsova, Shock waves in Lifshitz-like spacetimes, JHEP 04 (2015) 011 [arXiv: 1410.4595] [INSPIRE].

[61] U. Gürsoy, M. Järvinen, G. Nijs and J.F. Pedraza, Inverse Anisotropic Catalysis in Holographic QCD, JHEP 04 (2019) 071 [Erratum ibid. 09 (2020) 059] [arXiv:1811.11724] [INSPIRE].

[62] M. Strickland, Thermalization and isotropization in heavy-ion collisions, Pramana 84 (2015) 671 [arXiv: 1312.2285] [INSPIRE].

[63] ALICE collaboration, Centrality dependence of the charged-particle multiplicity density at midrapidity in $\mathrm{Pb}-\mathrm{Pb}$ collisions at $\sqrt{s_{\mathrm{NN}}}=5.02 \mathrm{TeV}$, Phys. Rev. Lett. 116 (2016) 222302 [arXiv: 1512.06104] [INSPIRE].

[64] I.Y. Aref'eva, A. Patrushev and P. Slepov, Holographic entanglement entropy in anisotropic background with confinement-deconfinement phase transition, JHEP 07 (2020) 043 [arXiv:2003.05847] [INSPIRE].

[65] D.S. Ageev, I.Y. Aref'eva, A.A. Golubtsova and E. Gourgoulhon, Thermalization of holographic Wilson loops in spacetimes with spatial anisotropy, Nucl. Phys. B 931 (2018) 506 [arXiv: 1606.03995] [INSPIRE].

[66] K. Bitaghsir Fadafan and R. Morad, Jets in a strongly coupled anisotropic plasma, Eur. Phys. J. C 78 (2018) 16 [arXiv:1710.06417] [InSPIRE].

[67] V. Skokov, A.Y. Illarionov and V. Toneev, Estimate of the magnetic field strength in heavy-ion collisions, Int. J. Mod. Phys. A 24 (2009) 5925 [arXiv:0907.1396] [InSPIRE].

[68] V. Voronyuk, V.D. Toneev, W. Cassing, E.L. Bratkovskaya, V.P. Konchakovski and S.A. Voloshin, (Electro-)Magnetic field evolution in relativistic heavy-ion collisions, Phys. Rev. C 83 (2011) 054911 [arXiv:1103.4239] [INSPIRE].

[69] A. Bzdak and V. Skokov, Event-by-event fluctuations of magnetic and electric fields in heavy ion collisions, Phys. Lett. B 710 (2012) 171 [arXiv:1111.1949] [INSPIRE].

[70] W.-T. Deng and X.-G. Huang, Event-by-event generation of electromagnetic fields in heavy-ion collisions, Phys. Rev. C 85 (2012) 044907 [arXiv:1201.5108] [InSPIRE].

[71] V. Toneev, O. Rogachevsky and V. Voronyuk, Evidence for creation of strong electromagnetic fields in relativistic heavy-ion collisions, Eur. Phys. J. A 52 (2016) 264 [arXiv: 1604.06231] [INSPIRE]. 
[72] S. Mereghetti, J. Pons and A. Melatos, Magnetars: Properties, Origin and Evolution, Space Sci. Rev. 191 (2015) 315 [arXiv: 1503.06313] [INSPIRE].

[73] R. Turolla, S. Zane and A. Watts, Magnetars: the physics behind observations. A review, Rept. Prog. Phys. 78 (2015) 116901 [arXiv: 1507.02924] [INSPIRE].

[74] K. Enqvist and P. Olesen, On primordial magnetic fields of electroweak origin, Phys. Lett. B 319 (1993) 178 [hep-ph/9308270] [INSPIRE].

[75] D. Grasso and H.R. Rubinstein, Magnetic fields in the early universe, Phys. Rept. 348 (2001) 163 [astro-ph/0009061] [INSPIRE].

[76] U. Gürsoy, I. Iatrakis, M. Järvinen and G. Nijs, Inverse Magnetic Catalysis from improved Holographic QCD in the Veneziano limit, JHEP 03 (2017) 053 [arXiv:1611.06339] [INSPIRE].

[77] U. Gürsoy, M. Jarvinen and G. Nijs, Holographic QCD in the Veneziano Limit at a Finite Magnetic Field and Chemical Potential, Phys. Rev. Lett. 120 (2018) 242002 [arXiv: 1707.00872] [INSPIRE].

[78] F.R. Brown et al., On the existence of a phase transition for QCD with three light quarks, Phys. Rev. Lett. 65 (1990) 2491 [INSPIRE].

[79] O. Philipsen and C. Pinke, The $N_{f}=2$ QCD chiral phase transition with Wilson fermions at zero and imaginary chemical potential, Phys. Rev. D 93 (2016) 114507 [arXiv:1602.06129] [INSPIRE].

[80] O. Philipsen, Constraining the phase diagram of QCD at finite temperature and density, PoS (LATTICE2019) 273 [arXiv: 1912 . 04827] [INSPIRE].

[81] M. D'Elia, S. Mukherjee and F. Sanfilippo, QCD Phase Transition in a Strong Magnetic Background, Phys. Rev. D 82 (2010) 051501 [arXiv: 1005.5365] [INSPIRE].

[82] E.M. Ilgenfritz, M. Kalinowski, M. Muller-Preussker, B. Petersson and A. Schreiber, Two-color QCD with staggered fermions at finite temperature under the influence of a magnetic field, Phys. Rev. D 85 (2012) 114504 [arXiv:1203.3360] [InSPIRE].

[83] M. D'Elia, Lattice QCD Simulations in External Background Fields, Lect. Notes Phys. 871 (2013) 181 [arXiv:1209.0374] [INSPIRE].

[84] L.D. Landau and E.M. Lifshits, Course of Theoretical Physics. Vol. 3: Quantum Mechanics: Non-Relativistic Theory, Pergamon, London U.K. (1958).

[85] F. Bigazzi, A. Caddeo, A.L. Cotrone and A. Paredes, Fate of false vacua in holographic first-order phase transitions, JHEP 12 (2020) 200 [arXiv: 2008.02579] [INSPIRE]. 